\title{
The Scaffolding Protein SYD-2/Liprin- $\alpha$ Regulates the Mobility and Polarized Distribution of Dense-Core Vesicles in C. elegans Motor Neurons
}

\author{
Patricia R. Goodwin ${ }^{1,2}$, Peter Juo ${ }^{1 *}$ \\ 1 Department of Molecular Physiology and Pharmacology, Sackler School of Graduate Biomedical Sciences, Tufts University School of Medicine, Boston, Massachusetts, \\ United States of America, 2 Graduate Program in Neuroscience, Sackler School of Graduate Biomedical Sciences, Tufts University School of Medicine, Boston,
} Massachusetts, United States of America

\begin{abstract}
The polarized trafficking of axonal and dendritic components is essential for the development and maintenance of neuronal structure and function. Neuropeptide-containing dense-core (DCVs) vesicles are trafficked in a polarized manner from the cell body to their sites of release; however, the molecules involved in this process are not well defined. Here we show that the scaffolding protein SYD-2/Liprin- $\alpha$ is required for the normal polarized localization of Venus-tagged neuropeptides to axons of cholinergic motor neurons in C. elegans. In syd-2 loss of function mutants, the normal polarized localization of INS22 neuropeptide-containing DCVs in motor neurons is disrupted, and DCVs accumulate in the cell body and dendrites. Time-lapse microscopy and kymograph analysis of mobile DCVs revealed that syd-2 mutants exhibit decreased numbers of DCVs moving in both anterograde and retrograde directions, and a corresponding increase in stationary DCVs in both axon commissures and dendrites. In addition, DCV run lengths and velocities were decreased in both axon commissures and dendrites of syd-2 mutants. This study shows that SYD-2 promotes bi-directional mobility of DCVs and identifies SYD-2 as a novel regulator of DCV trafficking and polarized distribution.
\end{abstract}

Citation: Goodwin PR, Juo P (2013) The Scaffolding Protein SYD-2/Liprin- $\alpha$ Regulates the Mobility and Polarized Distribution of Dense-Core Vesicles in C. elegans Motor Neurons. PLoS ONE 8(1): e54763. doi:10.1371/journal.pone.0054763

Editor: Brian D. McCabe, Columbia University, United States of America

Received September 26, 2012; Accepted December 14, 2012; Published January 24, 2013

Copyright: ( $) 2013$ Goodwin, Juo. This is an open-access article distributed under the terms of the Creative Commons Attribution License, which permits unrestricted use, distribution, and reproduction in any medium, provided the original author and source are credited.

Funding: This research was supported by a grant from the National Institutes of Health (NIH) to PJ (NS059953), a Basil O'Connor March of Dimes Scholar Award to PJ, and the Tufts Center for Neuroscience Research (P30 NS047243). PRG was supported by a Dean's Fellowship and the Synapse Neurobiology Training Program (T32 NS061764). The funders had no role in study design, data collection and analysis, decision to publish, or preparation of the manuscript.

Competing Interests: The authors have declared that no competing interests exist.

*E-mail: peter.juo@tufts.edu

\section{Introduction}

There are many similarities between $\mathrm{DCVs}$ and synaptic vesicles $(\mathrm{SVs})$. For example, both DCVs and $\mathrm{SVs}$ can be localized in a polarized manner at presynaptic sites and are transported to axons by the kinesin UNC-104/KIF1A in C. elegans, Drosophila and rodent cultured neurons [1-6]. Recent studies in C. elegans motor neurons showed that the polarized trafficking of both $\mathrm{SVs}$ and DCVs are regulated by cyclin-dependent kinase CDK-5 which functions to prevent inappropriate trafficking of these vesicles to dendrites [7,8]. Despite these similarities, SVs and DCVs also have distinct properties. For example, SVs are filled with chemical neurotransmitters and are typically clustered and released at active zones $[9,10]$. In contrast, DCVs contain neuropeptides, neurotrophins and peptide hormones, which are involved in modulating synaptic transmission and plasticity [11-13]. DCVs are excluded from active zones [14], and exocytosis of DCVs typically requires higher frequency stimulation for release than SVs [15,16]. After release, $\mathrm{SV}_{\mathrm{s}}$ can be refilled with neurotransmitter at synaptic terminals and undergo multiple rounds of exocytosis and endocytosis. In contrast, DCVs are not recycled in this manner, and must be replenished by newly generated DCVs from the cell body. DCVs are packaged with their protein cargo at the transgolgi network and undergo a complex biogenesis process, prior to being transported from the cell body to their sites of release [17-
21]. While several studies have focused on understanding how SVs are trafficked and localized to presynaptic sites [8,22-30], the molecules that mediate the targeting of DCVs to their release sites in axons are not well understood. Given that DCVs have different trafficking requirements and are more broadly distributed at release sites than $\mathrm{SVs}$, it is not clear if previously defined regulators of SV trafficking and clustering are also required to localize DCVs to their release sites.

Here we investigated whether SYD-2/Liprin- $\alpha$ regulates the polarized trafficking of DCVs to axons. SYD-2/Liprin- $\alpha$ is a multifunctional scaffolding protein that localizes to the active zone and is required for presynaptic terminal differentiation in $C$. elegans and Drosophila [31-35]. SYD-2/Liprin- $\alpha$ can oligomerize and interact with other presynaptic components to regulate active zone assembly and morphology in C. elegans and Drosophila [31,32,36-41]. For example, in HSN neurons, active zone components and SVs fail to localize at presynaptic sites and instead mis-localize to non-synaptic sites in the axon of syd-2 mutants [36,37].

SYD-2/Liprin- $\alpha$ has also been implicated in regulating intracellular transport. SYD-2/Liprin- $\alpha$ has been shown to interact with kinesin motors and regulate the movement characteristics of those motors and their associated cargo [42-45]. For example, in Drosophila, Liprin- $\alpha$ associates with kinesin-1 and promotes the anterograde flux and run length of its SV cargo [43]. In C. elegans, 
SYD-2 binds and clusters kinesin UNC-104/KIF1A, and promotes its anterograde flux and velocity [45]. Mammalian SYD-2/Liprin- $\alpha$ has been shown to bind UNC-104/KIF1A in vitro but the function of this interaction in mammalian neurons has not been studied [44]. Finally, Liprin- $\alpha$ can associate with the PDZ scaffold GRIP1 and promote the trafficking of postsynaptic proteins such as glutamate receptors in cultured rodent hippocampal neurons [46-49]. These studies show that in addition to promoting synapse formation, SYD-2 can interact with motors and regulate the trafficking of motors and multiple types of cargo.

A recent synaptic profiling study implicated SYD-2 as a regulator of DCV localization. This systematic study compared the protein composition of motor neuron synapses across a panel of genetic mutant backgrounds and showed that syd-2 mutants had decreased amounts of the neuropeptide INS-22 at presynaptic sites [50], however, the reason for this decrease is not known. Here, we investigate the mechanism by which SYD-2 affects the abundance of neuropeptides in motor neuron axons. We confirm that SYD-2 regulates the abundance of INS-22 at presynaptic sites and show that SYD-2 also affects the levels of another neuropeptide NLP-21 in axons. We use quantitative fluorescence analysis to examine the distribution of these Venus-tagged neuropeptides in motor neuron axons and dendrites and show that SYD-2 is required for the normal polarized distribution of DCVs to axons. Furthermore, time-lapse microscopy of mobile $\mathrm{DCVs}$ in motor neurons reveals that SYD-2 promotes the overall mobility of DCVs in axon commissures and dendrites. This study identifies SYD-2 as a novel regulator of DCV trafficking and polarized distribution.

\section{Materials and Methods}

\section{Strains}

Strains were maintained on OP50 E. coli at $20^{\circ} \mathrm{C}$ as described by Brenner et al. (1974) [51]. The following strains were used in this study: N2 Bristol, nuIs195 (Punc-129::ins-22::venus), nuIs183(Punc129::nlp-12::Venus); nuIs168(Punc-129::rab-3::Venus), yuEx46(Punc-129::unc-9::gfp) (gift from Lars Dreier, University of California, Los Angeles), pzEx141(Punc-129::syd-2), syd-2(ju37), syd2(ok217), cdk-5(gm336), dhc-1(js319). The ju37 mutation in syd-2 is a point mutation which results in a premature stop codon in the coiled-coil domain (glutamine 397)(Zhen 1999) and a truncated protein [45]. The $o k 217$ mutation in $s y d-2$ is a deletion of the $\mathrm{N}$ terminal coiled-coil domains, which results in an ochre stop codon and no detectable protein product [45].

\section{Constructs, Transgenes, and Germline Transformation}

Plasmids were generated using standard cloning techniques and details are available upon request. Briefly, genomic syd-2 (gift from Mei Zhen, University of Toronto) was tagged at the N-terminus with mRFP using engineered Not I sites and subcloned under the control of the Punc-129 promoter to create Punc-129::mRFP::syd-2 (KP\#1132). The mRFP was subcloned from KP\#1132 (gift from Dave Simon and Josh Kaplan) to create Punc-129::syd-2 (FJ\#82). FJ\#82 was injected at $25 \mathrm{ng} / \mu \mathrm{l}$ together with a coinjection marker (Pmyo-2::NLS-mCherry) to create $p z E x 141$.

\section{Fluorescent Microscopy and Quantification}

All imaging was performed using a Zeiss M1 Axioimager microscope. For all static imaging experiments, young adult animals were immobilized with $30 \mathrm{mg} / \mathrm{mL}$ 2,3-Butanedione monoxamine (Sigma Aldrich) for 5-7 minutes and mounted on $2 \%$ agarose pads prior to imaging. Images were captured using a Zeiss 100X PlanApo objective (NA 1.4) and an Orca-ER (Hamamatsu) CCD camera. Maximum intensity projections were generated from Z-series stacks and line scans of fluorescent puncta were acquired using Metamorph (v7.1) software (Molecular Devices). For quantitative analyses of fluorescent ventral and dorsal nerve cord puncta, maximum intensity projections of $\mathrm{Z}$ series stacks ( $1 \mu \mathrm{m}$ total depth) were generated. Exposure settings and gain were selected to fill the 12-bit dynamic range to avoid saturation and were kept constant for all images of a given fluorescent marker. Because the fluorescent neuropeptide markers were much dimmer in the $\mathrm{VNC}$, we had to use different acquisition settings to image the VNG and DNC. Dorsal nerve cord (axon) images were taken using $1 \times 1$ binning and $50 \times$ gain, while ventral nerve cord (dendrite) images were taken using $2 \times 2$ binning and $100 \times$ gain. Consequently, the axonal and dendritic images cannot be directly compared. FluoSphere fluorescent beads (Invitrogen) were imaged every day to correct for variation in microscope light bulb intensity. All images were acquired from animals with their VNG or DNC oriented towards the objective. Laterally-oriented animals were excluded from analysis.

The unc-129 promoter drives expression of genes in DA motorneurons, which have processes projecting towards the head, and DB neurons, whose processes project towards the tail. DA6 is the most posterior DA neuron with visible expression of fluorescent markers driven by the unc-129 promoter [7]. Thus, all fluorescent signal posterior to the DA6 neuron cell body is derived only from $\mathrm{DB}$ neurons. To isolate signal originating from only DB axons in the DNC, we drew line scans along the DNG posterior to the site of entry of the DA6 commissure. To isolate signal originating from only $\mathrm{DB}$ dendrites in the $\mathrm{VNC}$, we drew line scans posterior to the DA6 cell body, as previously described [7].

Line scans of $\mathrm{VNC}$ and DNG puncta were obtained using MetaMorph (v6.0) and were subsequently analyzed in Igor Pro (v5) using custom written software, as previously described [52]. Puncta intensity and density were calculated from each image. Puncta intensity is calculated as the fractional increase in peak fluorescence of each puncta over fluorescent bead intensity for that imaging day. Puncta density is calculated as the average number of puncta per $10 \mu \mathrm{m}$ of VNC or DNC. Puncta density measurements of DCVs in the VNC dendrites likely overestimate the density of DCVs, especially in wild type animals where dendritic DGVs are very sparse, or in some cases, absent. Our analysis software requires at least two puncta to demarcate the region to be analyzed and ensure the cord is in focus. Thus, VNG images with fewer than two in focus DCVs were discarded from analysis.

Images of INS-22::Venus in DB6 cell bodies were acquired from animals with their lateral or ventral sides oriented towards the objective, and $\mathrm{Z}$ stacks were taken to a depth of $2 \mu \mathrm{m}$. The average fluorescence of three fluorescent patches per cell body was measured using Metamorph software, then corrected for daily bead values and analyzed in Microsoft Excel.

To quantify the fluorescence of INS-22::Venus in coelomocytes, we imaged a posterior coelomocyte near the dorsal nerve cord between the DA6 and DB7 cell bodies. Z-stacks were taken to a depth of $2 \mu \mathrm{m}$ and the average fluorescence of four fluorescent patches per cell body was measured using Metamorph software, then corrected for daily bead values and analyzed in Microsoft Excel, as previously described [53]. Changes in puncta intensity and density were analyzed for statistical significance using Student's t test for two genotype comparisons and Tukey-Kramer for multiple genotype comparisons.

\section{Time-lapse Microscopy}

Young adult worms were paralyzed in $360 \mu \mathrm{g} / \mathrm{mL}$ Levamisol (Sigma) dissolved in M9 buffer for 7 to 9 minutes prior to time- 
A

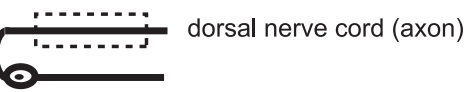

Axonal INS-22::Venus

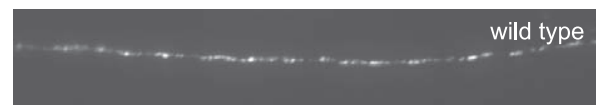

syd-2(ok217)
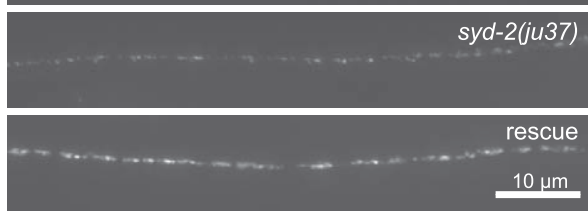

B Axonal Puncta

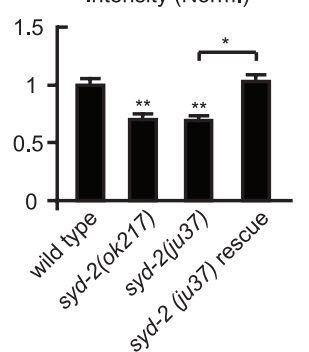

C Axonal Puncta Density (per $10 \mu \mathrm{m})$

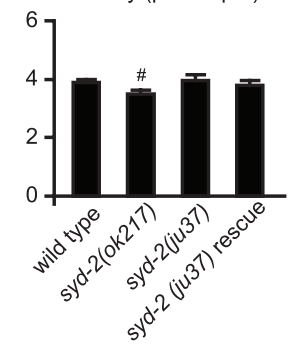

G

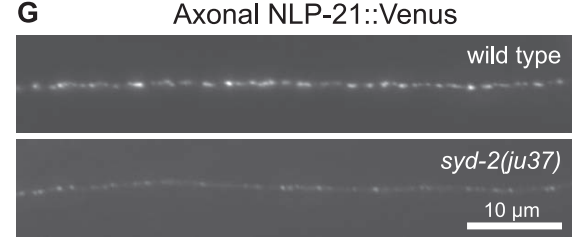

H

Axonal Puncta Intensity (Norm.)

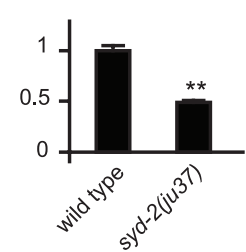

$\mathbf{M}$

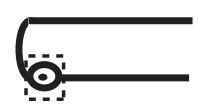

cell body
I Axonal Puncta Density (per $10 \mu \mathrm{m}$ )

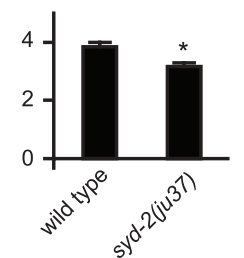

N Cell Body INS-22::Venus
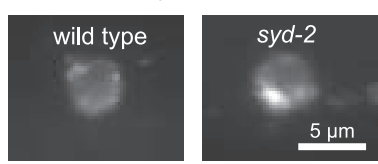

Cell Body Puncta

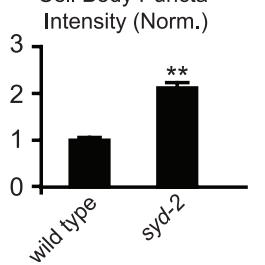

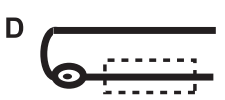

entral nerve cord (dendrite)

Dendritic INS-22::Venus

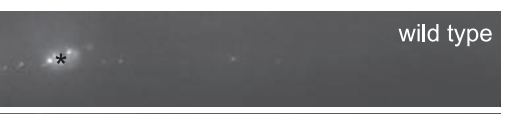

syd-2(ok217)

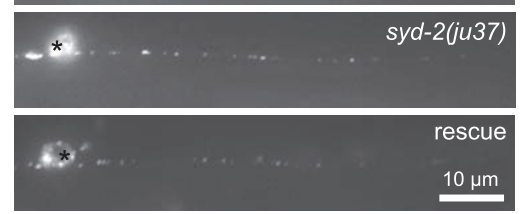

E Dendritic Puncta

F Dendritic Puncta Intensity (Norm.)
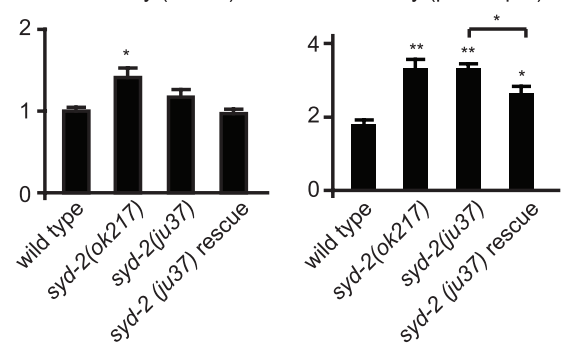

J Dendritic NLP-21::Venus

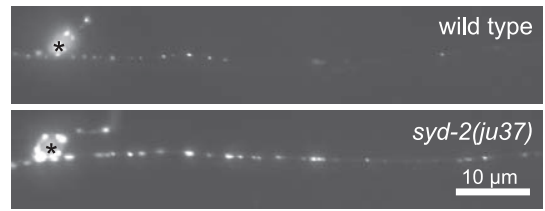

K Dendritic Puncta Intensity (Norm.)

L Dendritic Puncta
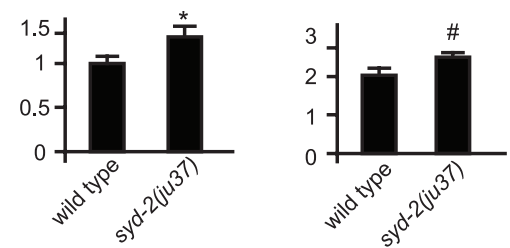

O Cell Body NLP-21::Venus

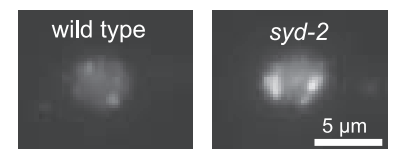

Cell Body Puncta
Intensity (Norm.)

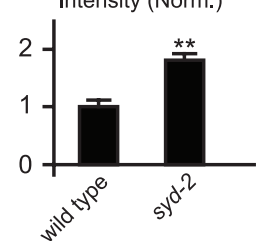

Figure 1. syd-2 regulates the polarized distribution of neuropeptides in DB motorneurons. (A) Schematic diagram of a DB motor neuron with its axon in the dorsal nerve cord (DNC) and its dendrite in the ventral nerve cord (VNC)(top panel). This diagram is oriented with the anterior of the animal to the left for this and all subsequent figures. The boxed region denotes that the axon was imaged for data presented in panels (A-C, $G-I)$. Representative images of INS-22::Venus in the axon of wild type, syd-2(ok217), syd-2(ju37), and Punc-129::syd-2;syd-2(ju37) rescue animals (bottom panels). (B-C) Quantification of INS-22::Venus puncta intensity (B) and density (C) in the axons of wild-type ( $\mathrm{n}=33$ ), syd-2(ok217)( $\mathrm{n}=24)$, syd2(ju37) $(\mathrm{n}=17)$, and syd-2 rescue animals $(\mathrm{n}=14)$. (D) Schematic diagram of DB motor neuron (top panel). The boxed region denotes that the dendrite was imaged for panels (D-F, J-L). Representative images of INS-22::Venus in the DB dendrite of wild-type, syd-2(ok217), syd-2(ju37), and Punc-129::syd- 
2;syd-2(ju37) rescue animals (bottom panel). (E-F) Quantification of INS-22::Venus puncta intensity (E) and density (F) in the dendrites of wild-type $(\mathrm{n}=44)$, syd-2(ok217)( $\mathrm{n}=31)$, syd-2(ju37)( $\mathrm{n}=30)$, and Punc-129::syd-2;syd-2(ju37) rescue animals $(\mathrm{n}=19)$. (G) Representative images of NLP-21::Venus in the DB axons of wild type and syd-2(ju37) mutant animals. (H-I) Quantification of NLP-21::Venus puncta intensity (H) and density (I) in the axons of wild type $(n=12)$ and syd-2(ju37) $(n=18)$ mutant animals. (J) Representative images of NLP-21::Venus in dendrites of wild type and syd-2(ju37) mutant animals. (K-L) Quantification of NLP-21::Venus puncta intensity (K) and density (L) in the dendrites of wild-type $(n=20)$ and syd-2(ju37)( $\mathrm{n}=22)$ animals. For this and all subsequent figures, error bars denote standard error from the mean (SEM) and cell bodies in the images are marked by an asterisk. Values that differ significantly (Tukey-Kramer (B, C, E and F); Student's $t$ test (H, I,K, and L) from wild type (marked by asterisks above each bar) or from other genotypes (comparisons marked by brackets) are denoted on the graphs (\#p $\leq 0.05,{ }^{*} p \leq 0.01,{ }^{* *} p \leq 0.001$ ). (M) Schematic diagram of a DB neuron. The boxed region denotes that the cell body was imaged for data presented in panels (N-O). (N) Representative images of INS-22::Venus in the motor neuron cell bodies of wild-type and syd-2(ok217) mutant animals. The graph shows quantification of somatic INS-22::Venus puncta fluorescence intensity for wild-type $(n=24)$ and syd-2(ok217) $(n=22)$ mutant animals. (O) Representative images of NLP-21::Venus in the motor neuron cell bodies of wild type and syd-2(ju37) mutant animals. The graph shows quantification of somatic NLP-21::Venus puncta fluorescence intensity for wild type $(n=7)$ and syd-2(ju37)( $(n=10)$ mutant animals. Values that differ significantly from wild type (Student's $t$ test) are denoted on the graphs $(* * p \leq 0.001)$.

doi:10.1371/journal.pone.0054763.g001

lapse. Animals were mounted on $2 \%$ agarose pads containing $360 \mu \mathrm{g} / \mathrm{ml}$ Levamisol. Time-lapse images of INS-22::Venus in dendrites were acquired from a region of the VNC between the DA6 and DB7 cell bodies. Because DB processes project towards the posterior, all movements towards the tail were designated as anterograde and all movements towards the head were designated as retrograde. Time-lapse imaging of DB6 axon commissures were acquired from animals with their ventral or lateral side oriented towards the objective. Line scans were drawn from the DB6 cell body to the last in-focus INS-22::Venus punctum in the commissure.

Time-lapse images were acquired at $4 \mathrm{~Hz}$ for 20 seconds and used to generate kymographs in MetaMorph (v7.1). Mobile INS-

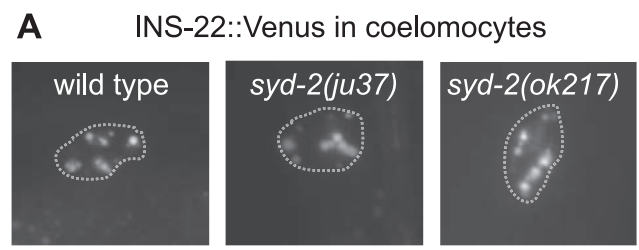

B Average INS-22::Venus Fluorescence Intensity in coelomocytes

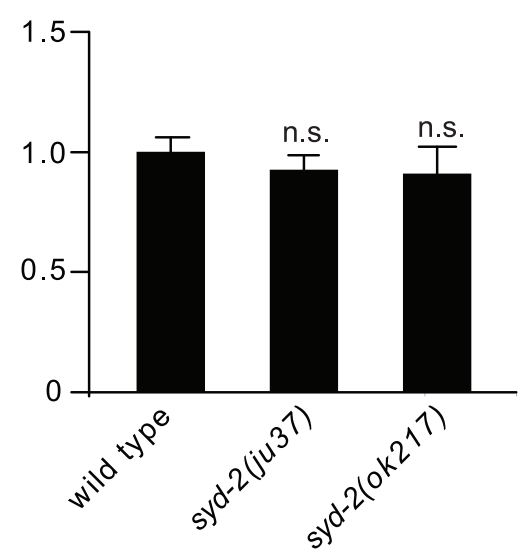

Figure 2. Analysis of INS-22::Venus accumulation in coelomocytes of syd-2 mutants. (A) Representative images of INS-22::Venus accumulation in coelomocytes (outlined by a dotted line) of wild type, syd-2(ju37) and syd-2(ok217) mutant animals. (B) Quantification of INS22::Venus fluorescence intensity in coelomocytes of wild type $(n=39)$, syd-2(ju37) $(\mathrm{n}=27)$ and syd-2(ok217)( $\mathrm{n}=14)$ mutant animals. Average intensity (normalized) \pm standard error are shown. Values that did not differ significantly from wild type (Student's $t$ test) are denoted on the graph by n.s. $(p>0.05)$.

doi:10.1371/journal.pone.0054763.g002
22::Venus puncta on these kymographs were traced in order to calculate direction of puncta movement and velocity. Each mobile punctum was traced during its longest uninterrupted period of movement. Puncta were defined as mobile if they moved distances greater than twice their own width and at velocities greater than $0.1 \mu \mathrm{m} / \mathrm{s}$. Puncta that changed direction were traced in both directions. Kymograph traces were analyzed using Microsoft Excel and histograms of puncta velocity were generated using IgorPro (v5). Average anterograde and retrograde velocity and the number of stationary, anterogradely, and retrogradely moving puncta were calculated for each kymograph (i.e., per worm) and these data were compiled for each genotype and analyzed in Microsoft Excel.

\section{Results}

\section{SYD-2 Regulates the Abundance of DCVs in Motor Neuron Axons}

We investigated the role of $s y d-2$ in DCV trafficking in DA and DB subclasses of cholinergic motor neurons. DA and DB motor neurons have a simple polarized morphology with a cell body and dendrite in the ventral nerve cord (VNC) and an axon that extends across the body (axon commissure) and along the dorsal nerve cord (DNG) where it makes en-passant synapses onto muscle cells [54]. DA neuron axons and dendrites extend from the cell body towards the anterior, while DB neuron processes extend towards the posterior of the animal. We used the unc-129 promoter to express fluorescently tagged proteins in a subset of $10 \mathrm{DA}$ and DB neurons as previously described [7](see Materials and Methods). In order to visualize DCVs in these neurons, we expressed Venustagged Insulin-like-protein-22 (INS-22::Venus) or Neuropeptidelike-protein-21 (NLP-21::Venus) under control of the unc-129 promoter $[4,53]$. Both INS-22 and NLP-21 regulate synaptic transmission at the neuromuscular junction [4]. Fluorescentlytagged versions of these neuropeptides have been widely used to study DCV trafficking in C. elegans [4,7,18,20,21,50,53]. In DA and DB motor neurons, INS-22::Venus and NLP-21::Venus have a polarized distribution where they are localized to presynaptic sites in the axon and are largely excluded from the dendrite [4,7]. Trafficking of both NLP-21 and INS-22 require the kinesin UNC104/KIF1A, as has been shown for endogenous neuropeptides and other DCV markers [1,2,4-6].

To determine if syd-2 regulates the polarized distribution of $\mathrm{DCVs}$, we used quantitative fluorescence microscopy to examine the localization of INS-22::Venus and NLP-21::Venus in DB neurons in two syd-2 loss-of-function mutants, ju37 and ok217. Both syd-2(ju37) and syd-2(ok217) alleles contain mutations that result in premature stop codons $[35,45]$ (see Materials and Methods). We quantified the puncta intensity and density of fluorescently-tagged markers using custom written software [7,52](see Materials and Methods). Because INS-22::Venus puncta 
A

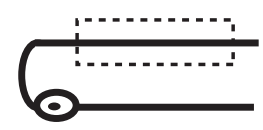

axon

Axonal INS-22::Venus

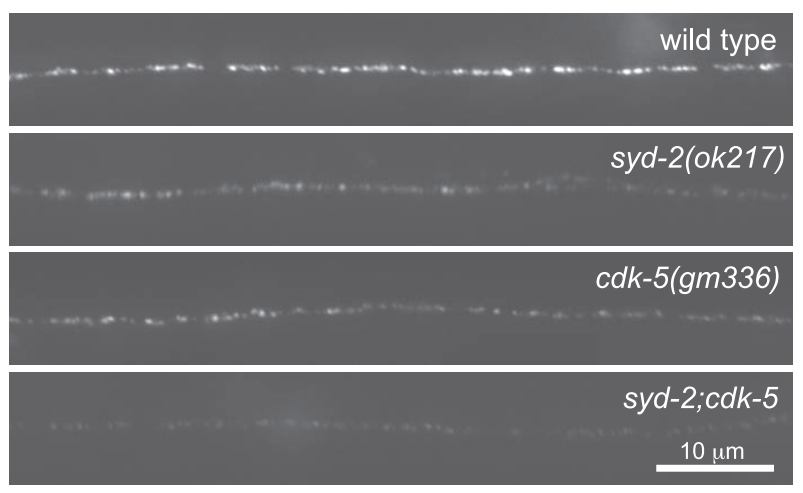

B

Axonal Puncta Intensity (Norm.)
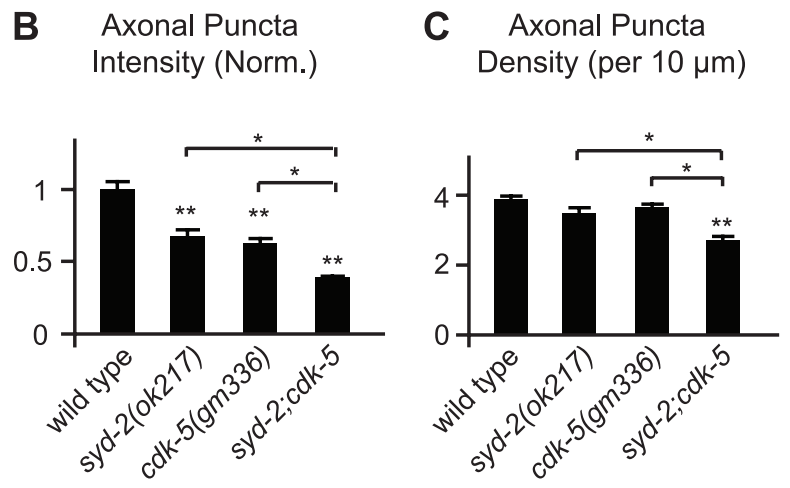

D
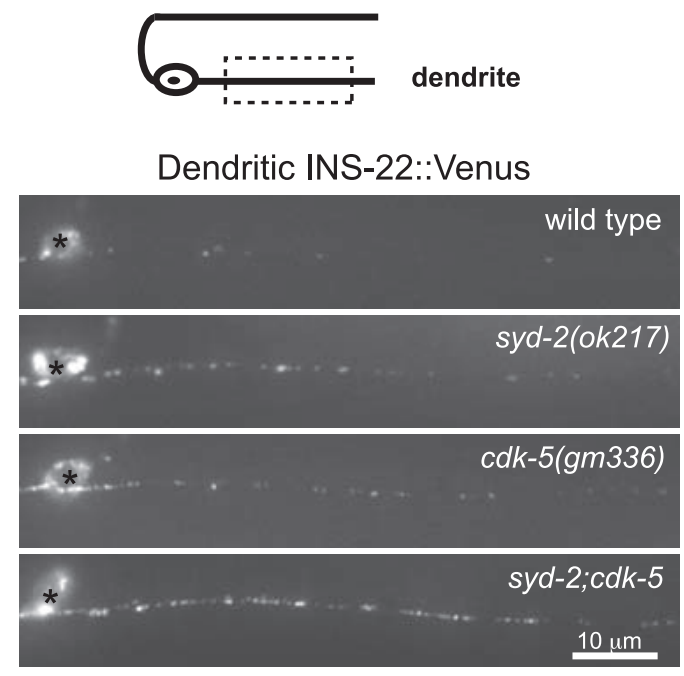
E Dendritic Puncta Intensity (Norm.)

\section{F Dendritic Puncta Density (per $10 \mu \mathrm{m}$ )}
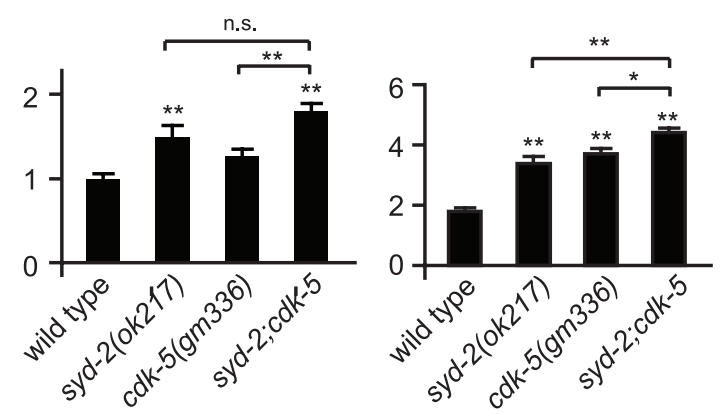

Figure 3. syd-2 and $c d k-5$ regulate the polarized distribution of neuropeptides through separate genetic pathways. (A) Schematic diagram of a DB motor neuron (top panel). The boxed region denotes that the axon was imaged for panels (A-C). Representative images of INS22::Venus in the axons of wild type, syd-2(ok217), cdk-5(gm336), and syd-2(ok217);cdk-5(gm336) double mutant animals (bottom panel). (B-C) Quantification of INS-22::Venus puncta intensity (B) and density (C) in the axons of wild type $(n=32)$, syd-2(ok217) $(n=24)$, cdk-5(gm336)( $n=35)$, and syd-2;cdk-5 double mutant $(\mathrm{n}=19)$ animals. (D) Schematic diagram of a DB motor neuron (top panel). The boxed region denotes that the dendrite was imaged for panels D-F. Representative images of INS-22::Venus in the dendrites of wild type, syd-2(ok217), cdk-5(gm336), and syd-2(ok217);cdk5(gm336) double mutant animals (bottom panel). (E-F) Quantification of INS-22::Venus puncta intensity (E) and density (F) in the dendrites of wild type $(\mathrm{n}=44)$, syd-2(ok217) $(\mathrm{n}=26), c d k-5(\mathrm{gm} 336)(\mathrm{n}=31)$, and syd-2;cdk-5 double mutant $(\mathrm{n}=32)$ animals. Values that differ significantly (Tukey-Kramer) from wild type (marked by asterisks above each bar) or from other genotypes (comparisons marked by brackets) are denoted on the graphs $\left(\# p \leq 0.05,{ }^{*} \mathrm{p} \leq 0.01,{ }^{* *} \mathrm{p} \leq 0.001\right)$. Values that did not differ significantly $(\mathrm{p}>0.05)$ are denoted by $\mathrm{n} . \mathrm{s}$. doi:10.1371/journal.pone.0054763.g003

can represent clusters of multiple DCVs [7], a change in the number of DCVs at a presynaptic site can result in changes in either puncta intensity or density. We found that syd-2(ju37) and syd-2(ok217) loss-of-function mutants had a significant reduction $(30 \%)$ in INS-22::Venus puncta fluorescence intensity in DB axons compared to wild type controls (Fig. 1A, B), consistent with previous studies using the ju37 allele [50]. Similarly, we found that the puncta fluorescence intensity of NLP-21::Venus was reduced by about $50 \%$ in syd-2(ju37) mutant axons (Fig. 1G, H). Analysis of INS-22::Venus and NLP-2 $1::$ Venus in syd-2 mutants also revealed that both neuropeptides had a small but significant decrease in puncta density in DB axons (Fig. 1C, I). However, the decrease in INS-22 density in axons did not reach statistical significance in all experiments (i.e., in the syd-2;cdk-5 double mutant analysis described below).

To determine if SYD-2 acts cell autonomously in DA/DB motor neurons to regulate neuropeptide abundance in axons, we performed cell-type specific rescue experiments. We expressed wild-type syd-2 cDNA under the control of the DA/DB motor neuron-specific promoter Punc-129 to restore SYD-2 function only in these motor neurons. Expression of $s y d-2$ in DA/DB neurons was sufficient to rescue the decrease in INS-22::Venus fluorescence in the axons of syd-2(ju37) mutants (Fig. 1A, B), indicating that SYD-2 functions in these cholinergic motor neurons to regulate neuropeptide abundance in axons.

Decreased INS-22::Venus fluorescence in the axon could result from either reduced trafficking of neuropeptides to presynaptic sites or enhanced release of neuropeptides from axons into the body cavity. Neuropeptide release can be indirectly measured in $C$. elegans by measuring the amount of fluorescently-tagged neuropeptides that accumulate in scavenger cells called coelomocytes, which endocytose secreted proteins from the body cavity [53]. We found no difference in the amount of INS-22::Venus in syd-2(ju37) and syd-2(ok217) mutant coelomocytes compared to wild type controls (Fig. 2), suggesting that increased release of neuropeptides is 
A

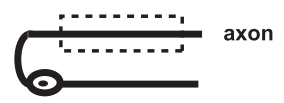

Axonal UNC-10/RIM-1::GFP
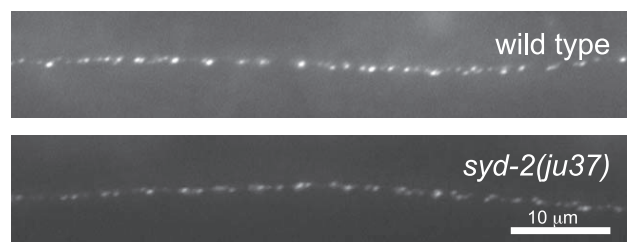

C Axonal RAB-3::Venus
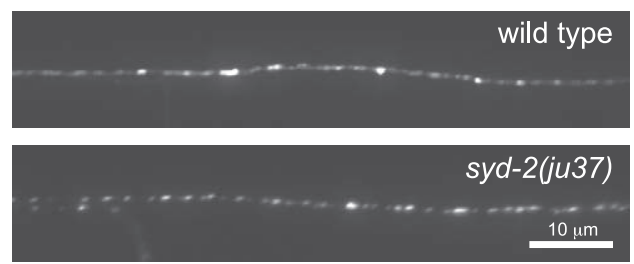

E Axonal UNC-9/Innexin::GFP

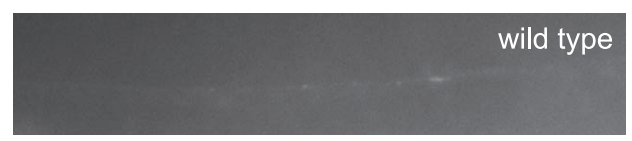

syd-2(ju37)

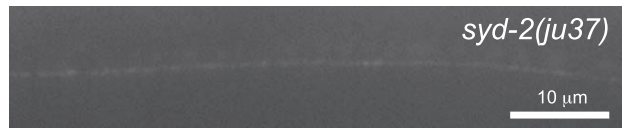

B

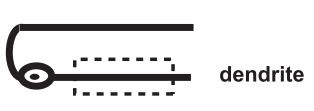

Dendritic UNC-10/RIM-1::GFP

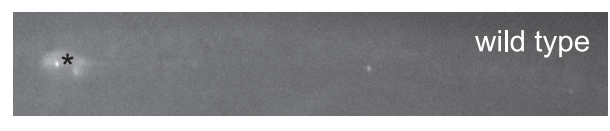

syd-2(ju37)

$10 \mu \mathrm{m}$

D Dendritic RAB-3::Venus

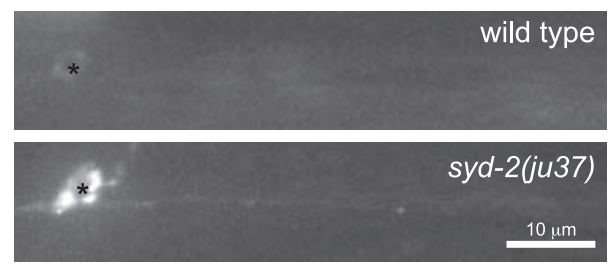

F Dendritic UNC-9/Innexin::GFP

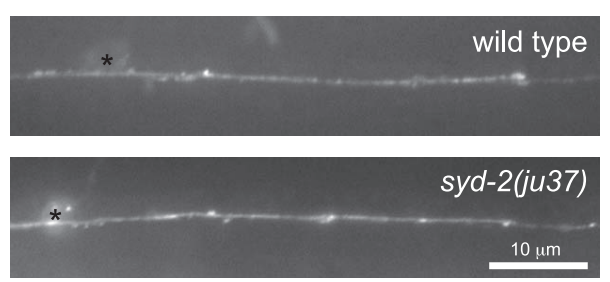

Figure 4. Analysis of axonal and dendritic markers in syd-2 mutants. (A) Schematic diagram of a DB motor neuron (top panel). The boxed region denotes that the axon was imaged in panels (A, C, E). Representative images of active zone protein UNC-10/Rim-1::GFP in the axons of wild type and syd-2(ju37) mutant animals (bottom panels). (B) Schematic diagram of a DB motor neuron (top panel). The boxed region denotes that the dendrite was imaged in panels (B, D, F). Representative images of active zone protein UNC-10/Rim-1::GFP in the dendrites of wild type and syd-2(ju37) mutant animals (bottom panels). (C) Representative images of synaptic vesicle protein RAB-3::Venus in the axons of wild type and syd-2(ju37) mutant animals (D) Representative images of synaptic vesicle protein RAB-3::Venus in the dendrites of wild type and syd-2(ju37) mutant animals. (E) Representative images of UNC-9/Innexin::GFP in the axons of wild type and syd-2(ju37) mutant animals. (F) Representative images of UNC-9/ Innexin::GFP in the dendrites of wild type and syd-2(ju37) mutant animals. doi:10.1371/journal.pone.0054763.g004

unlikely to account for the decrease in INS-22::Venus in syd-2 mutant axons.

\section{SYD-2 Regulates the Polarized Distribution of DCVs in DB Motor Neurons}

SYD-2 promotes the anterograde trafficking of synaptic vesicles in axons in Drosophila and C. elegans [43,45]. To determine if DCV trafficking is also regulated by SYD-2, we examined DB motor neuron cell bodies and dendrites for accumulation of Venustagged neuropeptides. We found that the amount of INS22::Venus and NLP-2 1::Venus fluorescence increased significantly in DB6 cell bodies of $s y d-2$ mutant animals relative to wild type controls (Fig. 1M-O), suggesting that syd-2 is required for trafficking of DCVs out of the cell body. We also found an increased abundance of DCVs in syd-2 mutant DB dendrites (Fig. 1D-F, J-L). Quantitative analysis of INS-22::Venus fluorescence in the VNC revealed an increase in INS-22::Venus density in both syd-2(ju37) and syd-2(ok217) mutant dendrites (Fig. 1D, F). syd-2(ok217) null mutants also had brighter INS-22::Venus puncta in the dendrite relative to wild type controls (Fig. 1D, E). We saw no significant change in INS-22::Venus puncta intensity in $s y d-$ 2(ju37) mutant dendrites (Fig. 1E). The weaker effect of the ju37 allele might suggest that $o k 217$ is a stronger syd-2 loss of function allele than ju37 (see Materials and Methods). We also found that
NLP-21::Venus fluorescence intensity and density were increased in the dendrites of syd-2(ju37) mutant animals relative to wild type controls (Fig. 1J-L). The increase in INS-22::Venus density observed in syd-2(ju37) mutant dendrites was partially rescued by expression of wild-type syd-2 cDNA under the control of the unc129 promoter (Fig. 1F). Together, these data indicate that SYD-2 functions in motor neurons to regulate the polarized distribution of DCVs to axons.

\section{SYD-2 and CDK-5 Function in Parallel Pathways to Regulate Polarized DCV Distribution}

We previously showed that cyclin-dependent kinase 5 (CDK-5) is required for polarized trafficking of DCVs to axons in DA/DB motor neurons [7]. Similar to syd-2 mutants, $c d k-5$ mutants exhibit a $40 \%$ reduction in INS-22::Venus puncta intensity in DB neuron axons and about an $80 \%$ increase in INS-22::Venus puncta density in DB neuron dendrites (Fig. 3) [7]. Since both $c d k-5$ and syd-2 mutants have similar defects in the polarized distribution of DCVs, we tested whether these two genes function in the same pathway by examining the distribution of INS-22::Venus in syd$2 ; c d k-5$ double mutants. If these two genes act in the same pathway, then the change in the polarized distribution of DCVs in syd-2;cdk-5 double mutants should be identical to either single mutant. However, in axons we found that syd-2;cdk-5 double 


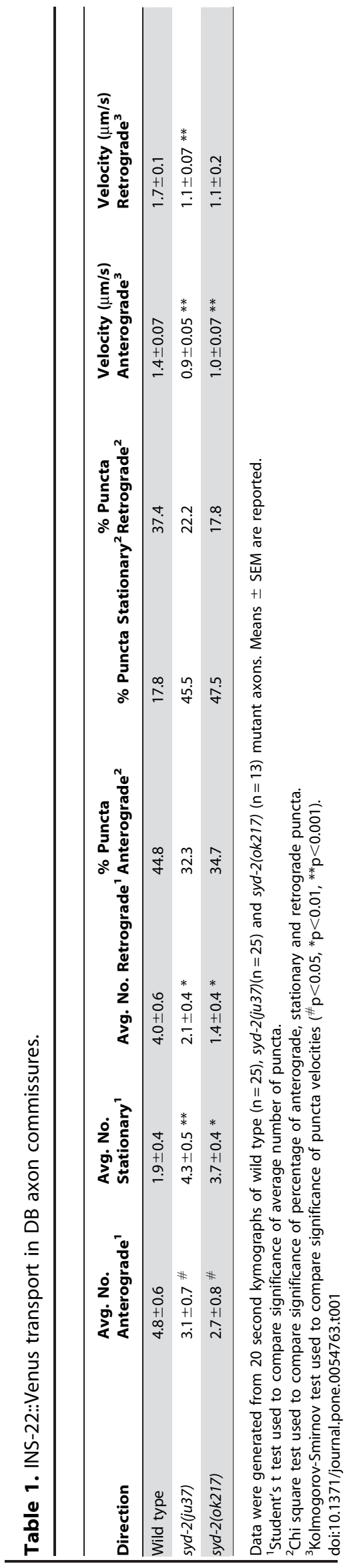

mutants had a dramatic decrease in INS-22::Venus puncta fluorescence compared to wild type controls and that this decrease was significantly different from either $s y d-2$ or $c d k-5$ single mutants (Fig. 3A-C). Similarly, in dendrites we found that syd-2;cdk-5 double mutants had an additive increase in INS-22::Venus puncta density compared to wild type controls and that this increase was significantly different from either syd-2 or $c d k-5$ single mutants (Fig. 3D, F). These additive effects suggest that SYD-2 functions in a novel, CDK-5-independent pathway to regulate the polarized distribution of DCVs.

\section{Axonal and Dendritic Markers are Relatively Normal in syd-2 Mutants}

Changes in the polarized distribution of DCVs observed in syd-2 mutants could be due to defects in synapse formation or neuronal polarity. Although SYD-2 is essential for active zone assembly and clustering of SVs at synapses in HSN neurons [36,37], the absolute requirement for SYD-2 for presynaptic differentiation may vary depending on the neuronal cell type $[35,50]$. In order to investigate the role of $s y d-2$ in synapse formation and neuronal polarity in DB neurons, we examined the distribution of two axonal markers, the active zone protein UNC-10/RIM-1::GFP and the synaptic vesicle-associated protein RAB-3::Venus $[4,55]$ in syd-2 mutants. In wild type animals, UNC-10::GFP and RAB$3::$ Venus (under control of the Punc-129 promoter) are localized to presynaptic sites in motor neuron axons but not dendrites (Figure 4A-D) $[4,7,26]$. We found that although syd-2 is not required for the localization of UNC-10::GFP or RAB-3::Venus to presynaptic sites in DB axons (Fig. 4A, G), we observed some mislocalization of UNC-10::GFP to dendrites (Fig. 4B) and increased accumulation of RAB-3::Venus in cell bodies (Fig. 4D) of $s y d-2$ mutants. These findings, together with the relatively normal density of DCVs in syd-2 mutant axons (Fig. 1), suggest that the number of presynaptic sites is not dramatically altered in syd-2 mutant DB neurons. These results are consistent with another study which showed that the fluorescence intensity and density of several presynaptic markers either did not change or decreased about $10-20 \%$ in DA/DB motor neuron axons of syd-2 mutants [50]. Taken together, these studies suggest that SYD-2 is not absolutely required for presynaptic differentiation in DB motor neurons and suggests that SYD-2 likely functions redundantly with other proteins to regulate active zone assembly in these neurons.

We also examined the distribution of a dendritic marker, the invertebrate GAP junction innexin protein UNG-9::GFP [26] in syd-2 mutants. We found that the normal dendritic localization of the innexin UNC-9::GFP in wild type motor neurons was unaltered in syd-2 mutants (Fig. 4E, F), suggesting that syd-2 mutants do not have a general defect in dendritic trafficking or identity. Together with our axonal marker analysis, these data suggest that syd-2 mutants do not have gross defects in synapse development or axon and dendrite development in DB motor neurons.

\section{syd-2 Regulates the Trafficking of DCVs in DB Axons and Dendrites}

Since SYD-2 has been implicated in motor-dependent trafficking, we next tested whether SYD-2 is required for the anterograde movement of DCVs in axons. SYD-2/Liprin- $\alpha$ binds to motors such as UNC-104 and regulates their velocity and movement properties $[42,44,45]$. SYD-2/Liprin- $\alpha$ also regulates the motility of motor cargos such as the run length and directionality of SVs $[43,45]$. We directly measured DCV movement using time-lapse microscopy and kymograph analysis of mobile DCVs in the DB6 
A

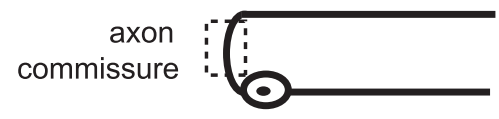

INS-22::Venus in DB6 axon commissure
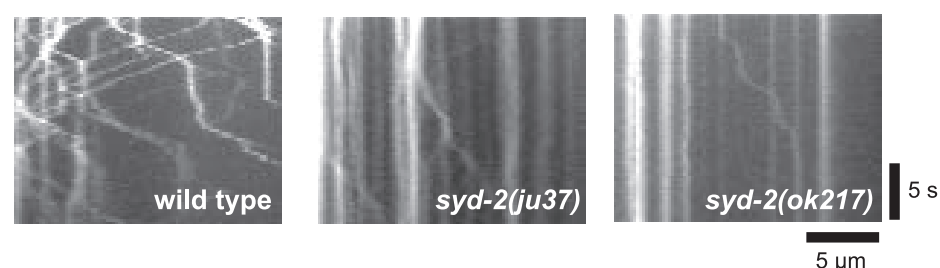

B

Direction of INS-22::Venus movement

in axon commissure

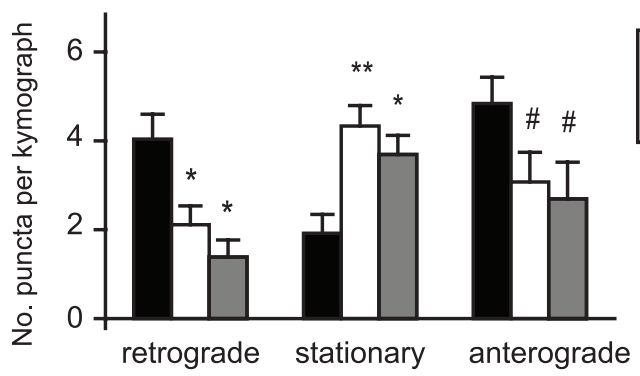

Mobile vs. stationary puncta in axon commissure (\%)

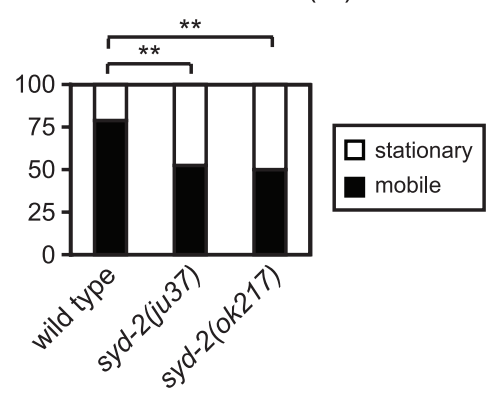

D Anterograde vs. retrograde movement in axon commissure (\%)

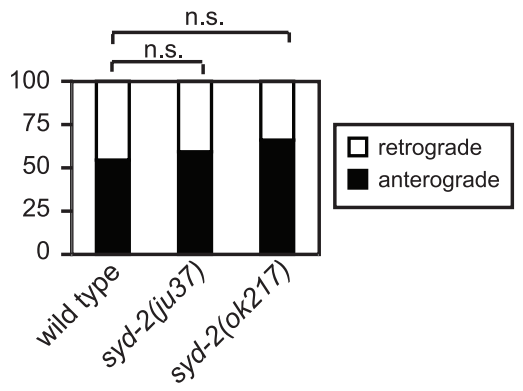

E INS-22::Venus velocity in axon commissure

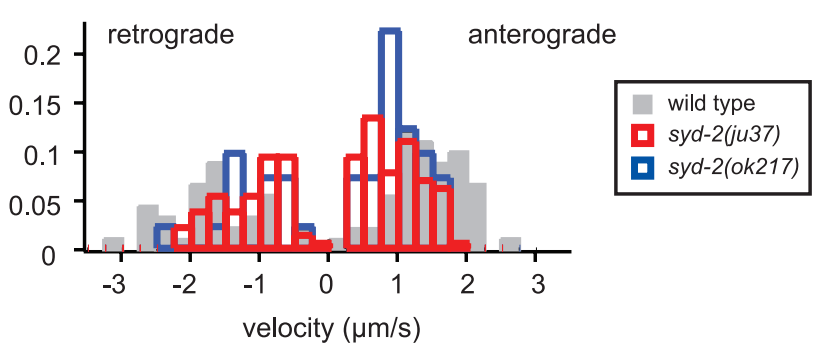

Figure 5. SYD-2 promotes the bi-directional movement of DCVs in the DB6 axon commissure. (A) Schematic diagram of a DB motor neuron (top panel). The boxed region denotes that the axon commissure was imaged for all the data presented in this figure. Representative kymographs from a $20 \mathrm{~s}$ movie of mobile INS-22::Venus puncta in the DB6 axon commissure of wild type, syd-2(ju37), and syd-2(ok217) mutant animals (bottom panels). These kymographs are oriented with the cell body and ventral side to the left and the dorsal side to the right. (B) Quantification of the average number of INS-22::Venus puncta moving anterogradely, retrogradely, or remaining stationary in each kymograph from wild type $(n=25)$, syd-2(ju37) $(n=25)$, and syd-2(ok217) $(n=13)$ animals. (C) Quantification of the percentage of mobile and stationary INS-22::Venus puncta in the DB6 axon commissure of wild type ( $n=227$ puncta), syd-2(ju37) ( $n=246$ puncta), and syd-2(ok217) $(n=96$ puncta) mutant animals. (D) Quantification of the percentage of anterograde and retrograde INS-22::Venus movement in the DB6 axon commissure of wild type ( $n=179$ puncta), syd-2(ju37)( $(\mathrm{n}=129$ puncta), and syd-2(ok217)( $\mathrm{n}=48$ puncta) mutant animals. (E) Histogram of INS-22::Venus puncta velocities in the axon commissures of wild type, syd-2(ju37), and syd-2(ok217) mutant animals. Positive values represent anterograde movements and negative values represent retrograde movements. Values that differ significantly (Student's $t$ test (B) and Chi Squared test (C-D)) from wild type are denoted on the graphs (\# $\left.p \leq 0.05,{ }^{*} p \leq 0.01,{ }^{* *} p \leq 0.001\right)$. Values that do not differ significantly $(p>0.05)$ are denoted by n.s. doi:10.1371/journal.pone.0054763.g005 


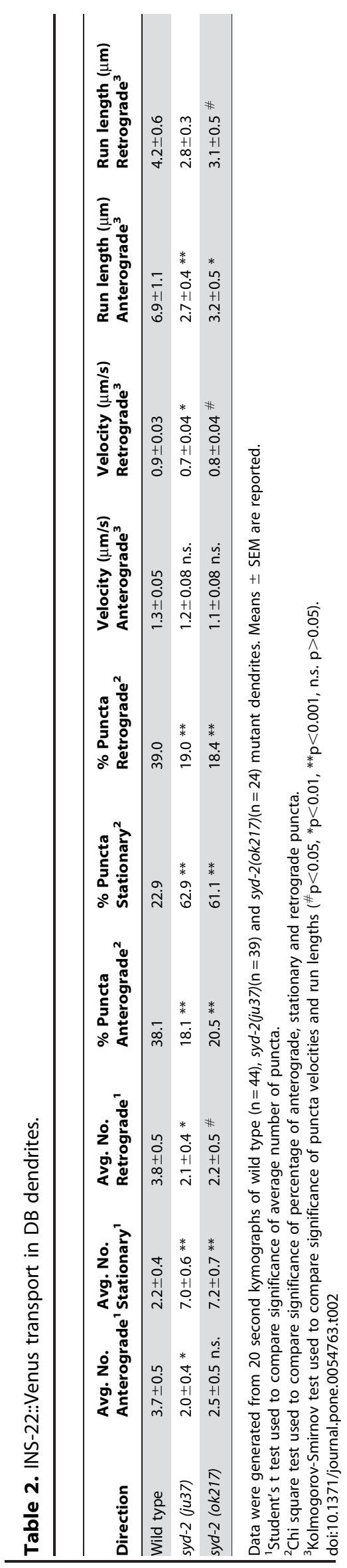

axon commissures of wild type and syd-2 mutants. The axon commissure is an asynaptic region of the axon connecting the cell body in the VNC to the presynaptic region of the axon in the DNG. Interestingly, we found that DCV trafficking in axon commissures was decreased in both the anterograde and retrograde directions in syd-2(ju37) and syd-2(ok217) mutant animals (Fig. 5A, B and Table 1). At the same time, we observed an increase in the number of stationary DGVs in the axon commissure in syd-2 mutants compared to wild type controls (Fig. 5 and Table 1). The percentage of mobile versus stationary DCVs was significantly decreased in syd-2 mutant axons (Fig. 5C and Table 1), whereas there was no change in the percentage of anterograde versus retrograde movement of DCVs (Fig. 5D and Table 1). Additionally, although we were unable to accurately measure run lengths in the axon commissure due to its curved trajectory, we found that DCV velocity was significantly reduced in both anterograde and retrograde directions in syd-2(ju37) and syd-2(ok217) mutant axons (Fig. 5E and Table 1). Because UNC104 is required to traffic DCVs to presynaptic sites in axons [2,4], these findings are consistent with reports that SYD-2 positively regulates the anterograde velocity of UNC-104 [45]. Interestingly, our data also show that SYD-2 regulates the velocity of DCVs moving in the retrograde direction, implicating SYD-2 as a positive regulator of bi-directional DCV trafficking.

Given that syd-2 mutants have reduced DCV movement in DB axon commissures and an accumulation of DCVs in dendrites, we next tested whether SYD-2 also affects dendritic DCV trafficking. Similar to our findings in the axon commissure, we observed a large increase in the number of stationary puncta in the dendrites of syd-2(ju37) and syd-2(ok217) mutant animals (Fig. 6A, $\mathrm{B}$ and Table 2). We also observed a decrease in the number of DCVs moving in the anterograde and retrograde directions in syd2 mutant dendrites, although the decrease in anterograde DCVs observed in the ok217 mutant allele did not reach statistical significance (Fig. 6B and Table 2). Similar to our findings in axons, we found that the percentage of mobile versus stationary DCVs was significantly reduced in syd-2(ju37) and syd-2(ok217) mutant dendrites (Fig. 6C), whereas the percentage of anterograde versus retrograde DCVs was not altered (Fig. 6D and Table 2). In addition, we found that syd-2 mutants exhibit a small decrease in retrograde DCV velocity and reduced DCV run lengths in both directions in DB dendrites (Table 2). Taken together, our data support a role for SYD-2 as a general positive regulator of DCV movement in axon commissures and dendrites.

syd-2 also Regulates DCV Mobility in DA Motor Neurons

Interestingly, a previous study showed that the mechanisms involved in regulating the polarized trafficking of SVs can differ between DA and DB classes of cholinergic motor neurons [8]. To determine if SYD-2 regulates DCV movement in DA as well as DB class motor neurons, we performed time-lapse imaging of mobile DCVs in the DA3 axon commissure. Similar to our results from DB6 neurons, we found that syd-2(ju37) mutants exhibit increased amounts of stationary DCVs and decreased bi-directional DCV movement in DA3 axon commissures (Fig. 7A). Likewise, we found that loss of syd-2 function reduced the percentage of mobile versus stationary DCVs (Fig. 7B), but not the ratio of anterograde to retrograde movement in DA3 axon commissures (ratio of anterograde/retrograde DCVs: wild type: 46\%/54\%; syd-2(ju37): 40\%/60\%). Anterograde and retrograde DCV velocities were also reduced in DA3 commissures of syd2(ju37) mutant animals (average anterograde velocity $(\mu \mathrm{m} / \mathrm{s})$ : wild type: $1.07 \pm 0.04$; syd-2(ju37): $0.69 \pm 0.08$; average retrograde velocity $(\mu \mathrm{m} / \mathrm{s})$ : wild type: $1.45 \pm 0.05$; syd-2(ju37): $0.91 \pm 0.07)$. 


\section{A}

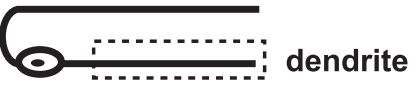

INS-22::Venus in dendrite

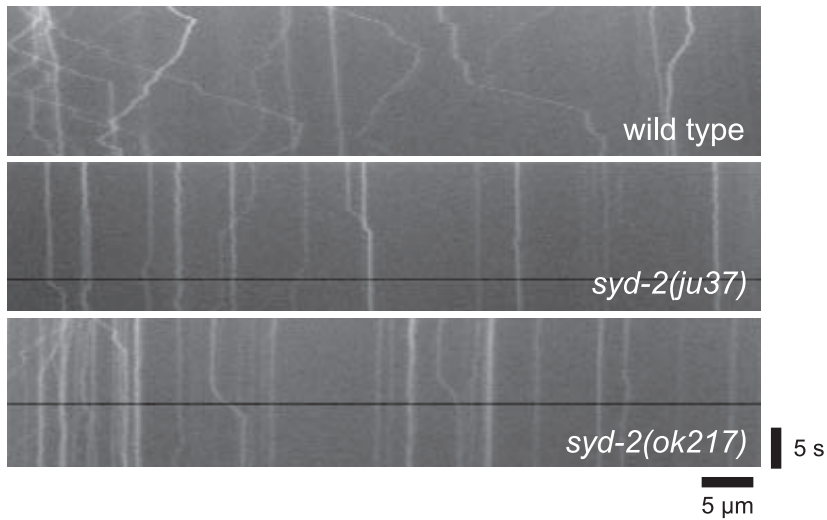

B Direction of INS-22::Venus movement

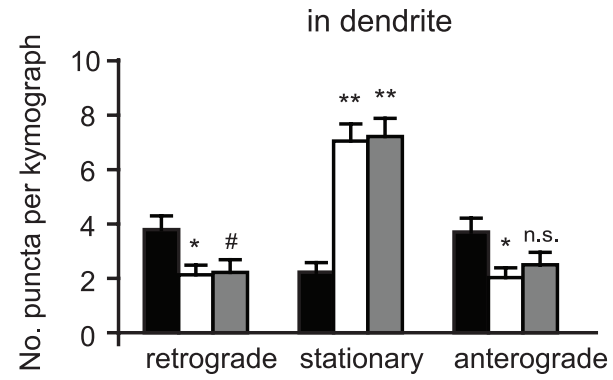

C Mobile vs. stationary puncta in dendrite (\%)

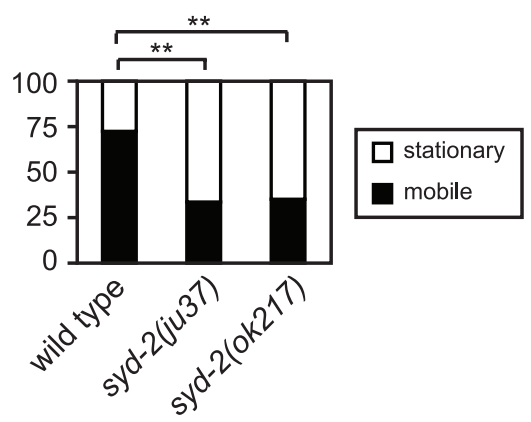

E

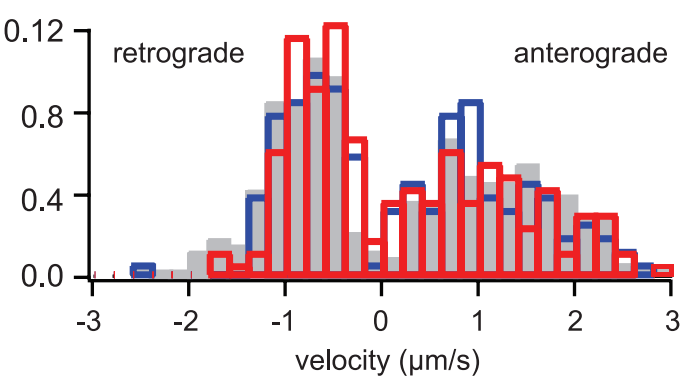

D Anterograde vs. retrograde movement in dendrite $(\%)$

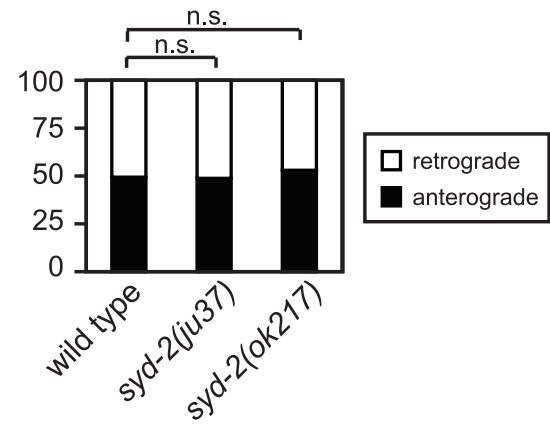

F INS-22::Venus run length

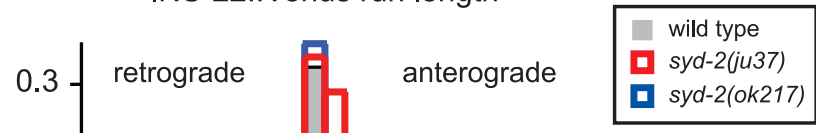

Figure 6. SYD-2 promotes the bi-directional movement of DCVs in DB dendrites. (A) Schematic diagram of a DB motor neuron (top panel). The boxed region denotes that the dendrite was imaged for all the data presented in this figure. Representative kymographs from a $20 \mathrm{~s}$ movie of mobile INS-22::Venus puncta in the DB dendrites of wild type, syd-2(ju37), and syd-2(ok217) mutant animals (bottom panels). (B) Quantification of the 
average number of INS-22::Venus puncta moving anterogradely, retrogradely, or remaining stationary in each kymograph from wild type $(\mathrm{n}=44)$, syd2(ju37) $(\mathrm{n}=39)$, and syd-2(ok217) $(\mathrm{n}=32)$ animals. (C) Quantification of the percentage of mobile and stationary INS-22:::Venus puncta in the DB dendrites of wild type ( $\mathrm{n}=355$ puncta), syd-2(ju37) ( $\mathrm{n}=414$ puncta), and syd-2(ok217) ( $\mathrm{n}=356$ puncta) mutant animals. (D) Quantification of the percentage of anterograde and retrograde INS-22::Venus movement in the DB dendrites of wild type $(n=257$ puncta), syd-2(ju37) ( $n=139$ puncta), and syd-2(ok217) ( $\mathrm{n}=125$ puncta) mutant animals. (E) Histogram of INS-22::Venus puncta velocities in the DB dendrites of wild type, syd-2(ju37), and syd-2(ok217) mutant animals. Positive values represent anterograde movements and negative values represent retrograde movements. (F) Histogram of INS-22::Venus puncta run length in DB dendrites. Positive values represent anterograde movements and negative values represent retrograde movements. Values that differ significantly (Student's $t$ test (B) and Chi Squared test (C-D)) from wild type are denoted on the graphs (\# $\mathrm{p} \leq 0.05$, $\left.{ }^{*} p \leq 0.01,{ }^{* *} p \leq 0.001\right)$. Values that do not differ significantly $(p>0.05)$ are denoted by n.s. doi:10.1371/journal.pone.0054763.g006

Although we are not able to measure INS-22::Venus signal solely in DA dendrites due to overlap with DB dendrites, we can enrich for INS-22::Venus signal in DA neurons by imaging a section of the VNC where the vast majority of fluorescence signal comes from DA3 dendrites. However, we acknowledge that overlapping DB3 dendrites may make a minor contribution to the
INS-22::Venus signal in this region due to occasional, weak expression of INS-22::Venus in DB3 neurons [7]. Time-lapse analysis of DCV movement in DA3 dendrites of syd-2 mutants revealed a significant decrease in the number of mobile DCVs, an increase in the number of stationary DCVs, and a corresponding decrease in the percentage of mobile versus stationary DCVs
A

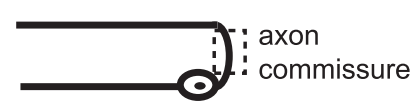

\section{Direction of INS-22::Venus movement in DA3 axon commissure}

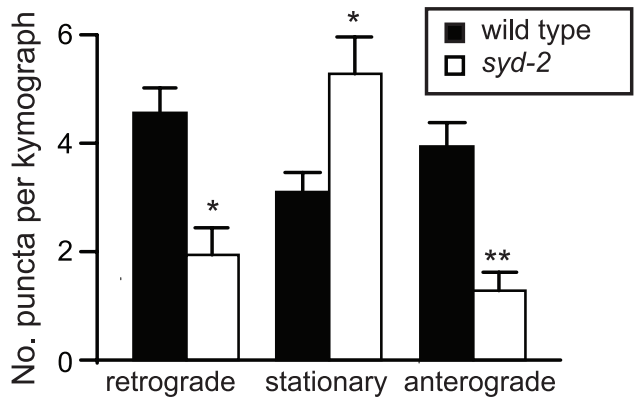

B Mobile vs stationary puncta in DA3 axon commissure (\%)

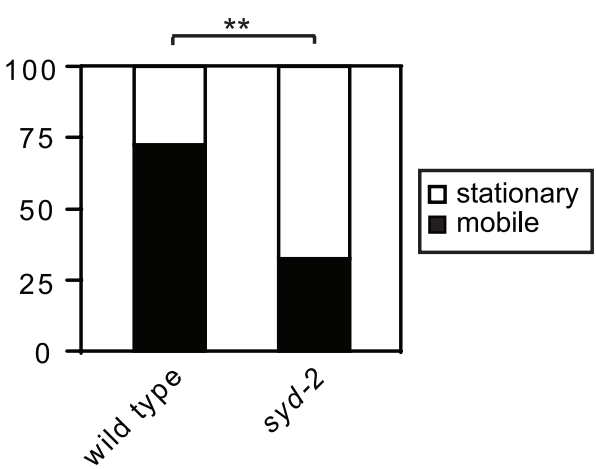

C<smiles>[Ge]=C1CCCCC1</smiles>

\section{Direction of INS-22::Venus movement in DA3 dendrite}

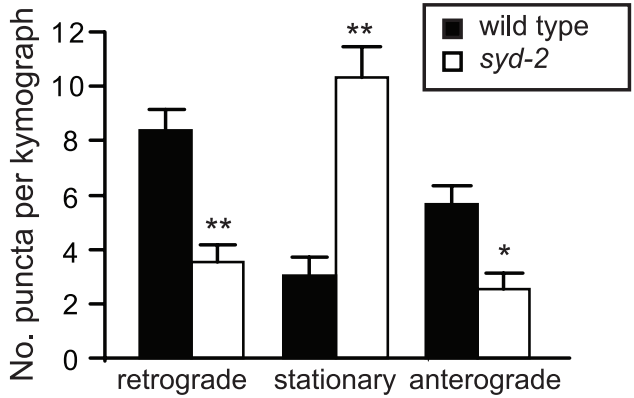

D Mobile vs stationary puncta in DA3 dendrite (\%)

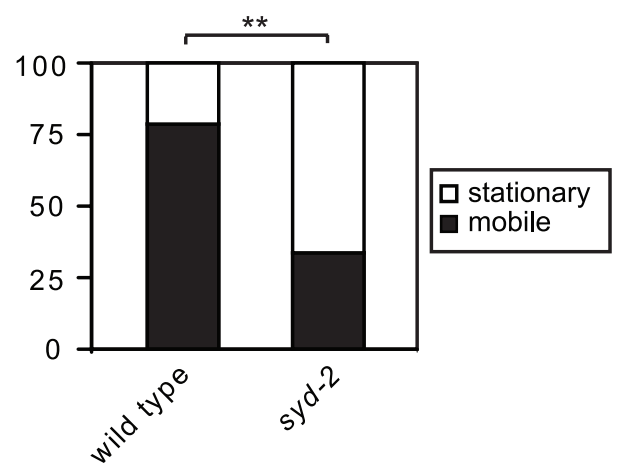

Figure 7. SYD-2 promotes the bi-directional movement of DCVs in DA3 axon commissures and dendrites. (A) Schematic diagram of a DA motor neuron (top panel). The boxed region denotes that the axon commissure was imaged for the data presented in panels (A-B). The graph (bottom panel) shows quantification of the average number of INS-22::Venus puncta moving anterogradely, retrogradely, or remaining stationary in kymographs generated from $20 \mathrm{~s}$ movies of mobile INS-22::Venus puncta in axon commissures of wild type $(n=29)$ and syd-2(ju37)( $\mathrm{n}=18)$ mutant animals. (B) Quantification of the percentage of mobile and stationary INS-22::Venus puncta in the DA3 axon commissure of wild type ( $\mathrm{n}=325$ puncta) and syd-2(ju37) ( $\mathrm{n}=141$ puncta) mutant animals. (C) Schematic diagram of a DA motor neuron (top panel). The boxed region denotes that the dendrite was imaged for the data presented in panels (C-D). The graph (bottom panel) shows quantification of the average number of INS22::Venus puncta moving anterogradely, retrogradely, or remaining stationary in kymographs generated from $20 \mathrm{~s}$ movies of mobile INS-22::Venus

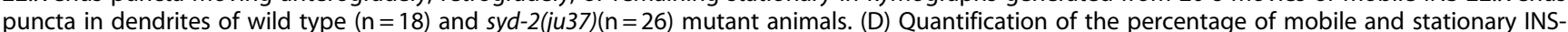
22::Venus puncta in the DA3 dendrite of wild type ( $n=257$ puncta) and syd-2(ju37) ( $n=405$ puncta) mutant animals. Values that differ significantly (Student's $t$ test $(A, C)$ and Chi Squared test $(B, D))$ from wild type are denoted on the graphs $\left(\# p \leq 0.05,{ }^{*} p \leq 0.01,{ }^{* *} p \leq 0.001\right)$.

doi:10.1371/journal.pone.0054763.g007 


\section{A}

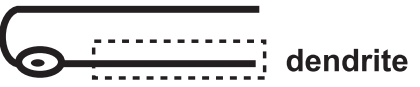

INS-22::Venus in dendrite

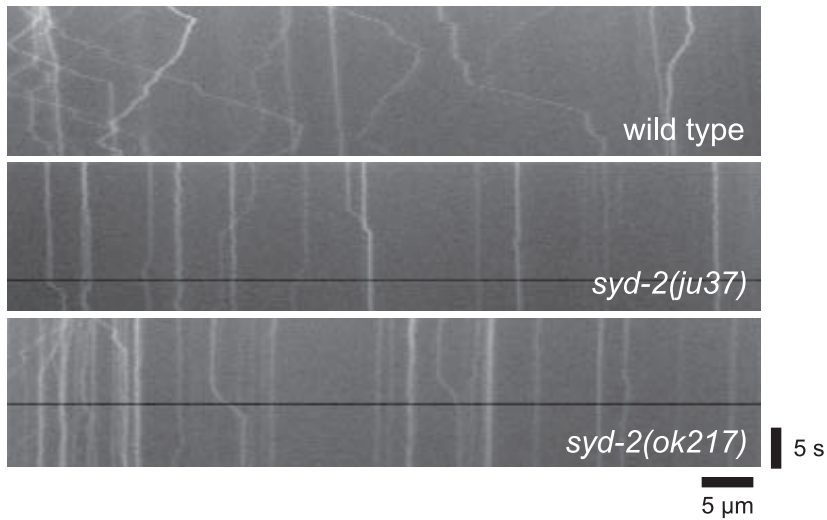

B Direction of INS-22::Venus movement

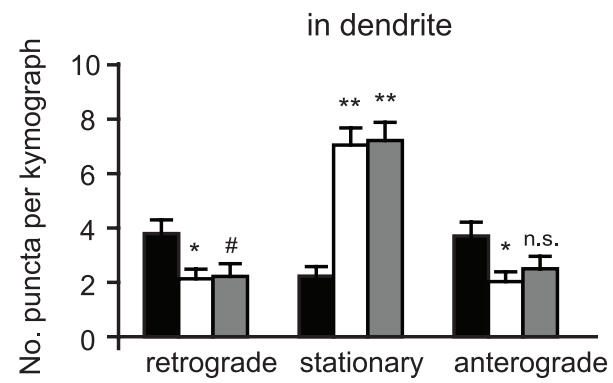

C Mobile vs. stationary puncta in dendrite (\%)

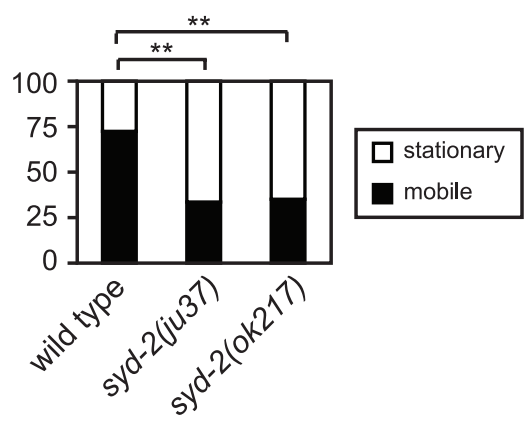

E

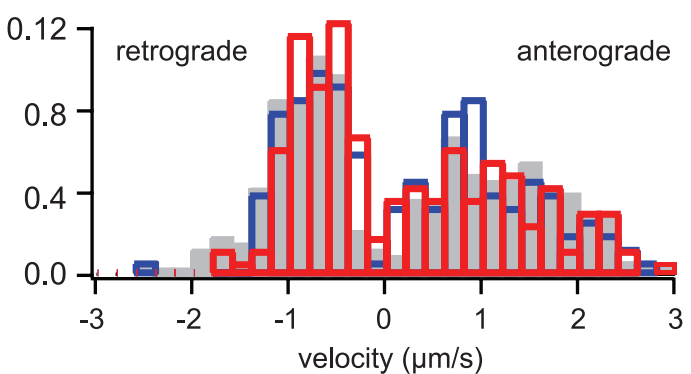

D Anterograde vs. retrograde movement in dendrite $(\%)$

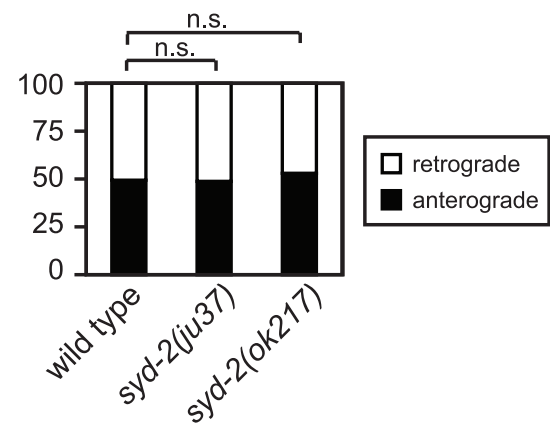

F INS-22::Venus run length

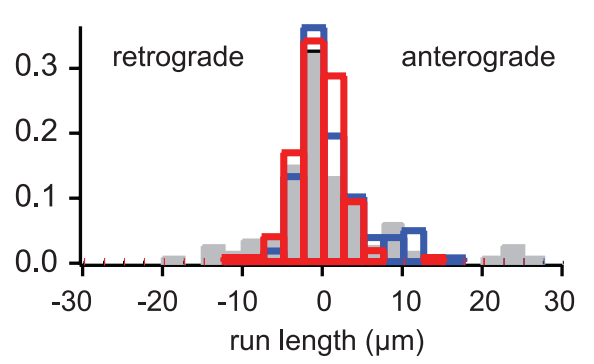

Figure 8. Dynein is required for the increase in dendritic DCVs in syd-2 mutants. (A) Schematic diagram of a DB motor neuron (top panel). The boxed region denotes that the dendrite was imaged. Representative images of INS-22::Venus in the dendrites of wild type, syd-2(ju37), dhc1(js319), and syd-2; dhc-1 mutant animals. (B) Quantification of INS-22::Venus puncta density in the dendrites of wild type $(n=44)$, syd-2(ju37) $(n=30)$, 
dhc-1(js319) $(n=12)$, and syd-2;dhc-1 $(n=16)$ mutant animals. Values that differ significantly (Tukey-Kramer) from wild type (marked by asterisks above each bar) or from other genotypes (comparisons marked by brackets) are denoted on the graphs (\#p $\left.\leq 0.05,{ }^{*} p \leq 0.01,{ }^{* *} p \leq 0.001\right)$. Values that did not differ significantly $(\mathrm{p}>0.05)$ are denoted by n.s. doi:10.1371/journal.pone.0054763.g008

(Fig. 7C, D). These results indicate that syd-2 mutants have similar defects in DCV mobility in both DA and DB motor neurons suggesting that SYD-2 promotes DCV trafficking in both axon commissures and dendrites in both motor neuron classes.

\section{Dynein is Required for Increased Dendritic DCVs in syd-2 Mutants}

Because syd-2 mutants have decreased bi-directional movement of DCVs in the dendrite, yet still manage to accumulate more stationary DCVs, we investigated whether active transport of $\mathrm{DGVs}$ on microtubules was required for the increase in dendritic DCVs observed in syd-2 mutants. We previously used EB1 plusend binding protein dynamics to show that approximately $90 \%$ of the microtubules in DB dendrites are oriented minus-end out relative to the cell body and the microtubule minus-end directed motor dynein is required for anterograde trafficking of DCVs into dendrites [7]. In order to test whether dynein-mediated transport is also required for the increased dendritic localization of DCVs in syd-2 mutants, we analyzed the distribution of INS-22::Venus in syd-2;dhc-1(js319) double mutant dendrites. We found that the $d h c^{-}$ 1 (js319) loss of function mutation blocked the increase in INS22::Venus puncta density observed in syd-2(ju37) mutants (Fig. 8). This result suggests that dynein is required for the increased accumulation of DCVs in $s y d-2$ mutant dendrites.

\section{Discussion}

\section{SYD-2 Regulates DCV Movement}

Neuropeptide-containing DCVs can be localized in a polarized manner to axons but the molecules involved are not well defined. Here, we show that the scaffolding protein SYD-2/Liprin- $\alpha$ is required for the normal polarized localization of $\mathrm{DCV}$ s to axons in cholinergic DA and DB motor neurons. The polarized localization of two DCV markers, neuropeptides INS-22::Venus and NLP$21::$ Venus, to axons is disrupted in $s y d-2$ loss of function mutants (Fig. 1). Time-lapse microscopy revealed that syd-2 mutants have several defects in DGV movement in DB motor neurons. We found that the number of DCVs moving in both the anterograde and retrograde directions is decreased in axon commissures and dendrites in two independent $s y d-2$ loss of function mutants (Figs. 5 and 6). This decrease in DCV movement was combined with a $2-$ 3 fold increase in the number of stationary DCVs resulting in a decrease in the percentage of mobile versus stationary DCVs in syd-2 mutants (Figs. 5 and 6). We also found that DCV velocities were reduced in syd-2 mutant axon commissures and dendrites and that DCV run lengths were decreased in syd-2 mutant dendrites (Tables 1 and 2). Finally, we found that the effects of syd2 on DCV trafficking were not specific to DB motor neurons, because we observed similar reductions in DCV movement in DA motor neurons (Fig. 7). These results indicate that SYD-2 promotes bi-directional DCV mobility in both axon commissures and dendrites of DA and DB motor neurons.

\section{Comparison of SYD-2 Effects on DCV and SV Trafficking}

Several studies have shown that SYD-2/Liprin- $\alpha$ can bind motors and regulate the movement of motors or their SV cargo [42-45]. Studies in Drosophila showed that Liprin- $\alpha$ interacts with the heavy chain of kinesin-1 in vitro and promotes the anterograde movement of $\mathrm{SVs}$ in axons [43]. Specifically, liprin- $\alpha$ mutants exhibit decreased anterograde flux of $\mathrm{SV}_{\mathrm{s}}$ in axons due to decreased anterograde processivity and increased initiation of retrograde movement [43]. In C. elegans, SYD-2 binds and clusters the SV motor UNC-104/KIF1A and promotes its anterograde movement and velocity, resulting in a decrease in net retrograde movement of SVs [45]. Our data show that SYD-2 promotes anterograde DCV trafficking and velocity in axon commissures of DB neurons (Fig. 5 and Table 1). Because UNC-104 traffics DCVs to presynaptic sites in DA/DB motor neuron axons [2,4,5,7], our data are consistent with the idea that SYD-2 promotes anterograde trafficking of DCVs on UNC-104 in axon commissures. Our data also show that SYD-2 promotes retrograde DCV trafficking in axon commissures (Fig. 5) revealing a novel role for SYD-2 in regulation of retrograde transport.

There are multiple ways in which SYD-2 could regulate the retrograde trafficking of DCVs. We previously showed that microtubule orientation is predominantly plus-end out relative to the cell body in axons and largely minus-end out in dendrites of DB motor neurons in C. elegans [7]. In the same study, we showed that the minus-end-directed microtubule motor dynein is required for anterograde trafficking of $\mathrm{DCVs}$ in these dendrites and likely also for retrograde trafficking in axons [7], consistent with a prior study showing that dynein transports $\mathrm{SVs}$ retrogradely in DA motor neuron axons [8]. These studies suggest that dynein transports DCVs retrogradely in axons and anterogradely in dendrites. Because we find that syd-2 mutants have reduced bidirectional trafficking of DCVs, our data could implicate SYD-2 in regulating dynein-mediated trafficking of DCVs. Consistent with this idea, we found that mutations in dynein heavy chain, $d h c^{-1}$, block the increased accumulation of DCVs in syd-2 mutant dendrites (Fig. 8). However, because a previous study failed to observe an interaction between SYD-2 and dynein [43], it remains possible that SYD-2 regulation of dynein is not direct.

SYD-2 might regulate retrograde trafficking of DCVs indirectly via its effects on anterograde trafficking. Multiple recent studies suggest that anterograde and retrograde movement of DCVs are tightly coupled. Mutation of the anterograde motor unc-104/ kinesin-3 disrupts both the anterograde and retrograde flux of DCVs in Drosophila [1]. Conversely, inhibition of the dynactin complex, which is required for dynein function, disrupts anterograde as well as retrograde trafficking [56]. Finally, by tracking individual DCVs throughout multiple sections of the axon, Wong et al (2012) demonstrated that DGVs undergo extensive longrange recirculation in axons, which may explain why disruption of DCV trafficking in one direction affects movement in the other direction [57].

Here we show that SYD-2 regulates two aspects of DCV trafficking: DCV mobility and the polarized localization of DCVs to axons versus dendrites. How loss of DCV mobility in syd-2 mutants could result in a loss of DCVs from presynaptic sites in the axon and a simultaneous increase in dendrites is not yet clear. One possible model is that in syd-2 mutants, the decrease in DCV mobility results in fewer DCVs reaching presynaptic sites due to an increase in stationary DCVs in the axon commissure and cell body. The accumulation of DCVs in the cell body might then lead to increased numbers of DCVs in the dendrite over time. This model is consistent with our previous data showing that in unc-104 mutants, DCVs are not trafficked to the axon but instead accumulate in the cell body and dendrite [7]. However, we 
cannot exclude the possibility that SYD-2 regulates the polarized localization of DCVs and bi-directional DCV trafficking through independent mechanisms. It remains possible that the change in the polarized distribution of INS-22::Venus in syd-2 mutants is due to compartment-specific changes in DCV release (i.e., more release from axons and less release from cell bodies and dendrites), however it has not yet been shown whether or not DCVs can be released from cell bodies and dendrites of DA/DB motor neurons. Further studies of DCV localization during development will be needed to determine if accumulation of DCVs in syd-2 mutant cell bodies precedes dendritic DCV accumulation and whether SYD2 's effects on DCV polarity are directly due to changes in DCV mobility.

Finally, SYD-2 may not directly regulate motor movement but might regulate the ability of DCVs to associate with motors. SYD2 has been proposed to function as an adaptor to link cargo to motors. For example, Liprin- $\alpha$ was shown to be degraded in a CaMKII-dependent manner in hippocampal neurons and this regulated degradation was proposed as a mechanism to promote cargo unloading at active synapses [47]. Intriguingly, CaMKII has been shown to promote the synaptic capture of trafficking DCVs in Drosophila neurons [58,59]. Our data are also consistent with a role for SYD-2 in promoting DCV attachment to motors. In this scenario, DCVs are unloaded prematurely in syd-2 mutant axons and dendrites, resulting in decreased run lengths and increased numbers of stationary DCVs. While this is an attractive model, the decreased DCV velocities observed in syd-2 mutants suggest that SYD-2 may also regulate the movement properties of DCV-motor complexes. It will be interesting in the future to test whether synaptic activity and CaMKII regulate SYD-2 degradation and DCV unloading from motors such as UNG-104 or dynein.

\section{Role of SYD-2 in Synapse Development Versus Intracellular Trafficking}

SYD-2/Liprin- $\alpha$ appears to have multiple functions in neurons. SYD-2/Liprin- $\alpha$ is essential for active zone assembly in C. elegans HSN neurons and regulates active zone morphology in C. elegans GABAergic neurons and at the Drosophila NMJ [31-33,35-37].

\section{References}

1. Barkus RV, Klyachko O, Horiuchi D, Dickson BJ, Saxton WM (2008) Identification of an axonal kinesin-3 motor for fast anterograde vesicle transport that facilitates retrograde transport of neuropeptides. Mol Biol Cell 19: 274-283.

2. Jacob TC, Kaplan JM (2003) The EGL-21 carboxypeptidase E facilitates acetylcholine release at Caenorhabditis elegans neuromuscular junctions. J Neurosci 23: 2122-2130.

3. Pack-Chung E, Kurshan PT, Dickman DK, Schwarz TL (2007) A Drosophila kinesin required for synaptic bouton formation and synaptic vesicle transport. Nat Neurosci 10: 980-989.

4. Sieburth D, Ch'ng Q, Dybbs M, Tavazoie M, Kennedy S, et al. (2005) Systematic analysis of genes required for synapse structure and function. Nature 436: 510-517.

5. Zahn TR, Angleson JK, MacMorris MA, Domke E, Hutton JF, et al. (2004) Dense core vesicle dynamics in Caenorhabditis elegans neurons and the role of kinesin UNC-104. Traffic 5: 544-559.

6. Lo KY, Kuzmin A, Unger SM, Petersen JD, Silverman MA (2011) KIF1A is the primary anterograde motor protein required for the axonal transport of densecore vesicles in cultured hippocampal neurons. Neurosci Lett 491: 168-173.

7. Goodwin PR, Sasaki JM, Juo P (2012) Cyclin-dependent kinase 5 regulates the polarized trafficking of neuropeptide-containing dense-core vesicles in Caenorhabditis elegans motor neurons. J Neurosci 32: 8158-8172.

8. Ou CY, Poon VY, Maeder CI, Watanabe S, Lehrman EK, et al. (2010) Two cyclin-dependent kinase pathways are essential for polarized trafficking of presynaptic components. Cell 141: 846-858.

9. Hammarlund M, Palfreyman MT, Watanabe S, Olsen S, Jorgensen EM (2007) Open syntaxin docks synaptic vesicles. PLOS Biol 5: e198.

10. Sudhof TC (2004) The synaptic vesicle cycle. Annu Rev Neurosci 27: 509-547.

11. Lessmann V, Brigadski T (2009) Mechanisms, locations, and kinetics of synaptic BDNF secretion: an update. Neurosci Res 65: 11-22.

12. Li C, Kim K (2008) Neuropeptides. WormBook: 1-36.
SYD-2/Liprin- $\alpha$ also interacts with microtubule motors and regulates intracellular trafficking of pre- and postsynaptic cargo $[43-45,48,49]$.

Although syd-2 plays an essential role in synapse formation in HSN neurons [36,37], studies in other neuronal cell types suggest that SYD-2 is not absolutely required for active zone assembly and clustering of SVs $[35,50]$, suggesting that in some cases SYD-2 functions with other molecules to regulate presynaptic development. While syd-2 does not appear to be required for clustering of active zone and SV markers in DA/DB motor neurons, we found a slight mislocalization of UNC-10::GFP to dendrites (Fig. 4) and Ch'ng et al. (2008) found a 20\% decrease in UNC-10::GFP fluorescence in axons suggesting that SYD-2 contributes to active zone assembly in these neurons [50]. However, loss of synaptic UNC-10 itself is unlikely to be a direct cause of DCV polarity defects because unc-10 loss of function mutants do not have defects in DCV localization [7]. Thus, although the effects of SYD-2 on synapse formation may contribute to DCV polarity defects in DB motor neurons, SYD-2 does not appear to play a major role in synapse formation in DB neurons. Instead, our data identify SYD2 as a novel regulator of bi-directional DCV mobility and the polarized distribution of DCVs. It will be interesting in the future to determine if SYD-2 regulates other motors and cargos and whether it plays a broader role in regulating intracellular transport.

\section{Acknowledgments}

We would like to thank Josh Kaplan, Mei Zhen, David Simon, Lars Dreier, Quee-Lim Ch'ng, and Derek Sieburth for strains, plasmids and reagents. We thank members of the Juo lab for helpful discussions and comments on this manuscript, and Jeremy Dittman and Dan Cox for data analysis software. We would also like to thank the Caenorhabditis elegans Genetics Center (CGG) for strains.

\section{Author Contributions}

Conceived and designed the experiments: PRG PJ. Performed the experiments: PRG. Analyzed the data: PRG PJ. Contributed reagents/ materials/analysis tools: PRG PJ. Wrote the paper: PRG PJ.

13. Park JJ, Loh YP (2008) How peptide hormone vesicles are transported to the secretion site for exocytosis. Mol Endocrinol 22: 2583-2595.

14. Hammarlund M, Watanabe S, Schuske K, Jorgensen EM (2008) CAPS and syntaxin dock dense core vesicles to the plasma membrane in neurons. J Cell Biol 180: 483-491.

15. Bruns D, Jahn R (1995) Real-time measurement of transmitter release from single synaptic vesicles. Nature 377: 62-65.

16. Verhage M, McMahon HT, Ghijsen WE, Boomsma F, Scholten G, et al. (1991) Differential release of amino acids, neuropeptides, and catecholamines from isolated nerve terminals. Neuron 6: 517-524.

17. Borgonovo B, Ouwendijk J, Solimena M (2006) Biogenesis of secretory granules. Curr Opin Cell Biol 18: 365-370.

18. Edwards SL, Charlie NK, Richmond JE, Hegermann J, Eimer S, et al. (2009) Impaired dense core vesicle maturation in Caenorhabditis elegans mutants lacking Rab2. J Cell Biol 186: 881-895.

19. Park JJ, Koshimizu H, Loh YP (2009) Biogenesis and transport of secretory granules to release site in neuroendocrine cells. J Mol Neurosci 37: 151-159.

20. Sumakovic M, Hegermann J, Luo L, Husson SJ, Schwarze K, et al. (2009) UNC-108/RAB-2 and its effector RIC-19 are involved in dense core vesicle maturation in Caenorhabditis elegans. J Cell Biol 186: 897-914.

21. Hannemann M, Sasidharan N, Hegermann J, Kutscher LM, Koenig S, et al. (2012) TBC-8, a putative RAB-2 GAP, regulates dense core vesicle maturation in Caenorhabditis elegans. PLOS Genet 8: e1002722.

22. Byrd DT, Kawasaki M, Walcoff M, Hisamoto N, Matsumoto K, et al. (2001) UNC-16, a JNK-signaling scaffold protein, regulates vesicle transport in C. elegans. Neuron 32: 787-800.

23. Crump JG, Zhen M, Jin Y, Bargmann CI (2001) The SAD-1 kinase regulates presynaptic vesicle clustering and axon termination. Neuron 29: 115-129. 
24. Hung W, Hwang C, Po MD, Zhen M (2007) Neuronal polarity is regulated by a direct interaction between a scaffolding protein, Neurabin, and a presynaptic SAD-1 kinase in Caenorhabditis elegans. Development 134: 237-249.

25. Park M, Watanabe S, Poon VY, Ou CY, Jorgensen EM, et al. (2011) CYY-1/ cyclin $\mathrm{Y}$ and CDK-5 differentially regulate synapse elimination and formation for rewiring neural circuits. Neuron 70: 742-757.

26. Poon VY, Klassen MP, Shen K (2008) UNC-6/netrin and its receptor UNC-5 locally exclude presynaptic components from dendrites. Nature 455: 669-673.

27. Sakaguchi-Nakashima A, Meir JY, Jin Y, Matsumoto K, Hisamoto N (2007) LRK-1, a C. elegans PARK8-related kinase, regulates axonal-dendritic polarity of SV proteins. Curr Biol 17: 592-598.

28. Sakamoto R, Byrd DT, Brown HM, Hisamoto N, Matsumoto K, et al. (2005) The Caenorhabditis elegans UNC-14 RUN domain protein binds to the kinesin1 and UNC-16 complex and regulates synaptic vesicle localization. Mol Biol Cell 16: $483-496$

29. Tanizawa Y, Kuhara A, Inada H, Kodama E, Mizuno T, et al. (2006) Inositol monophosphatase regulates localization of synaptic components and behavior in the mature nervous system of C. elegans. Genes Dev 20: 3296-3310.

30. West AE, Neve RL, Buckley KM (1997) Targeting of the synaptic vesicle protein synaptobrevin in the axon of cultured hippocampal neurons: evidence for two distinct sorting steps. J Cell Biol 139: 917-927.

31. Fouquet W, Owald D, Wichmann C, Mertel S, Depner H, et al. (2009) Maturation of active zone assembly by Drosophila Bruchpilot. J Cell Biol 186: $129-145$.

32. Kaufmann N, DeProto J, Ranjan R, Wan H, Van Vactor D (2002) Drosophila liprin-alpha and the receptor phosphatase Dlar control synapse morphogenesis. Neuron 34: 27-38.

33. Stigloher C, Zhan H, Zhen M, Richmond J, Bessereau JL (2011) The presynaptic dense projection of the Caenorhabditis elegans cholinergic neuromuscular junction localizes synaptic vesicles at the active zone through SYD-2/liprin and UNC-10/RIM-dependent interactions. J Neurosci 31: 43884396.

34. Yeh E, Kawano T, Weimer RM, Bessereau JL, Zhen M (2005) Identification of genes involved in synaptogenesis using a fluorescent active zone marker in Caenorhabditis elegans. J Neurosci 25: 3833-3841.

35. Zhen M, Jin Y (1999) The liprin protein SYD-2 regulates the differentiation of presynaptic termini in C. elegans. Nature 401: 371-375.

36. Dai Y, Taru H, Deken SL, Grill B, Ackley B, et al. (2006) SYD-2 Liprin-alpha organizes presynaptic active zone formation through ELKS. Nat Neurosci 9: 1479-1487.

37. Patel MR, Lehrman EK, Poon VY, Crump JG, Zhen M, et al. (2006) Hierarchical assembly of presynaptic components in defined C. elegans synapses. Nat Neurosci 9: 1488-1498.

38. Astigarraga S, Hofmeyer K, Farajian R, Treisman JE (2010) Three Drosophila liprins interact to control synapse formation. J Neurosci 30: 15358-15368.

39. Patel MR, Shen K (2009) RSY-1 is a local inhibitor of presynaptic assembly in C. elegans. Science 323: 1500-1503.

40. Taru H, Jin Y (2011) The Liprin homology domain is essential for the homomeric interaction of SYD-2/Liprin-alpha protein in presynaptic assembly. J Neurosci 31: 16261-16268.

41. Chia PH, Patel MR, Shen K (2012) NAB-1 instructs synapse assembly by linking adhesion molecules and F-actin to active zone proteins. Nat Neurosci 15: 234 242.
42. Hsu CC, Moncaleano JD, Wagner OI (2011) Sub-cellular distribution of UNC104(KIF1A) upon binding to adaptors as UNG-16(JIP3), DNG-1(DCTN1/ Glued) and SYD-2(Liprin-alpha) in C. elegans neurons. Neuroscience 176: 39 52.

43. Miller KE, DeProto J, Kaufmann N, Patel BN, Duckworth A, et al. (2005) Direct observation demonstrates that Liprin-alpha is required for trafficking of synaptic vesicles. Curr Biol 15: 684-689.

44. Shin H, Wyszynski M, Huh KH, Valtschanoff JG, Lee JR, et al. (2003) Association of the kinesin motor KIF1A with the multimodular protein liprinalpha. J Biol Chem 278: 11393-11401.

45. Wagner OI, Esposito A, Kohler B, Chen CW, Shen CP, et al. (2009) Synaptic scaffolding protein SYD-2 clusters and activates kinesin-3 UNC-104 in C. elegans. Proc Natl Acad Sci U S A 106: 19605-19610.

46. Dunah AW, Hueske E, Wyszynski M, Hoogenraad CC, Jaworski J, et al. (2005) LAR receptor protein tyrosine phosphatases in the development and maintenance of excitatory synapses. Nat Neurosci 8: 458-467.

47. Hoogenraad CC, Feliu-Mojer MI, Spangler SA, Milstein AD, Dunah AW, et al. (2007) Liprinalphal degradation by calcium/calmodulin-dependent protein kinase II regulates LAR receptor tyrosine phosphatase distribution and dendrite development. Dev Cell 12: 587-602.

48. Ko J, Kim S, Valtschanoff JG, Shin H, Lee JR, et al. (2003) Interaction between liprin-alpha and GIT1 is required for AMPA receptor targeting. J Neurosci 23: 1667-1677.

49. Wyszynski M, Kim E, Dunah AW, Passafaro M, Valtschanoff JG, et al. (2002) Interaction between GRIP and liprin-alpha/SYD2 is required for AMPA receptor targeting. Neuron 34: 39-52.

50. Ch'ng Q, Sieburth D, Kaplan JM (2008) Profiling synaptic proteins identifies regulators of insulin secretion and lifespan. PLOS Genet 4: e1000283.

51. Brenner S (1974) The genetics of Caenorhabditis elegans. Genetics 77: 71-94.

52. Burbea M, Dreier L, Dittman JS, Grunwald ME, Kaplan JM (2002) Ubiquitin and AP180 regulate the abundance of GLR-1 glutamate receptors at postsynaptic elements in C. elegans. Neuron 35: 107-120.

53. Sieburth D, Madison JM, Kaplan JM (2007) PKC-1 regulates secretion of neuropeptides. Nat Neurosci 10: 49-57.

54. White JG, Southgate E, Thomson JN, Brenner S (1986) The structure of the nervous system of Caenorhabditis elegans. Philos Trans R Soc Lond B Biol Sci 314: 1-340.

55. Koushika SP, Richmond JE, Hadwiger G, Weimer RM, Jorgensen EM, et al. (2001) A post-docking role for active zone protein Rim. Nat Neurosci 4: 997 1005.

56. Kwinter DM, Lo K, Mafi P, Silverman MA (2009) Dynactin regulates bidirectional transport of dense-core vesicles in the axon and dendrites of cultured hippocampal neurons. Neuroscience 162: 1001-1010.

57. Wong MY, Zhou C, Shakiryanova D, Lloyd TE, Deitcher DL, et al. (2012) Neuropeptide delivery to synapses by long-range vesicle circulation and sporadic capture. Cell 148: 1029-1038.

58. Shakiryanova D, Tully A, Levitan ES (2006) Activity-dependent synaptic capture of transiting peptidergic vesicles. Nat Neurosci 9: 896-900.

59. Wong MY, Shakiryanova D, Levitan ES (2009) Presynaptic ryanodine receptorCamKII signaling is required for activity-dependent capture of transiting vesicles. J Mol Neurosci 37: 146-150. 